\title{
ORIGINAL ARTICLE SUMOylation and SENP3 regulate STAT3 activation in head and neck cancer
}

\author{
Z Zhou ${ }^{1,2}, \mathrm{M} \mathrm{Wang}^{1}, \mathrm{~J} \mathrm{Li}^{2}, \mathrm{M} \mathrm{Xiao^{3 }}, \mathrm{YE} \mathrm{Chin}{ }^{3}, \mathrm{~J} \mathrm{Cheng}^{1}, \mathrm{ETH} \mathrm{Yeh}^{4}, \mathrm{~J} \mathrm{Yang}^{1}$ and $\mathrm{J} \mathrm{Yi}^{1}$
}

\begin{abstract}
Hyperphosphorylation of signal transducer and activator of transcription 3 (STAT3) has been found in various types of human cancers, including head and neck cancer (HNC). Although smoking is critical in the development and progression of HNC, how tobacco components activate STAT3 is unclear. We demonstrated that exposure of HNC cell lines to a tobacco extract induced a rapid Y705 phosphorylation of STAT3 and a rapid increase in the SUMO protease SENP3 that depended on a simultaneous increase in reactive oxygen species. We identified that SUMOylation at the lysine 451 site facilitated STAT3 binding to the phosphatase TC45 through an SUMO-interacting motif of TC45. SENP3 could thus enhance STAT3 phosphorylation by de-conjugating the SUMO2/3 modification of STAT3. Knocking-down of SENP3 greatly impaired basal and induced STAT3 phosphorylation by tobacco extract or interleukin 6. A correlation between SENP3 protein levels and STAT3 Y705 phosphorylation levels in human laryngeal carcinoma specimens was found, which was more significant in the specimens derived from the smoker patients and with poor clinicopathological parameters. Our data identified SUMOylation as a previously undescribed post-translational modification of STAT3 and SENP3 as a critical positive modulator of tobacco- or cytokine-induced STAT3 activation. These findings provide novel insights into the hyperphosphorylation of STAT3 in development of HNC.
\end{abstract}

Oncogene (2016) 35, 5826-5838; doi:10.1038/onc.2016.124; published online 16 May 2016

\section{INTRODUCTION}

Signal transducer and activator of transcription 3 (STAT3) is one of the critical mediators of cytokine signaling. Upon ligand-induced receptor dimerization and Janus kinases (JAKs) activation, STAT3 becomes phosphorylated on tyrosine or serine residues, which induces their dimerization, nuclear translocation and DNA binding, leading to the induction of cytokine-responsive genes. ${ }^{1,2}$ Aberrant sustained activation of STAT3 is found in various types of human malignancies. ${ }^{3-9}$ STAT3 is typically activated by the interleukin 6 (IL-6) family of cytokines or growth factors that can be constitutively and excessively secreted by epithelial tumor cells, stromal cells or immune cells. Phosphorylated STAT3 in turn activates target genes involved in cell survival, proliferation, differentiation and apoptosis. Thus, STAT3 mediates the communication between cancers and their microenvironments. ${ }^{10-13}$ STAT3 mutations are rare in human tumors. ${ }^{14,15}$ To date, regulatory mechanisms operating at the post-translational ${ }^{1,16,17}$ and epigenetic levels ${ }^{15}$ as well as protein-protein interactions ${ }^{18-20}$ have provided explanations for the hyperphosphorylation of STAT3 in cancers. However, it remains elusive whether the cancer microenvironment can stimulate STAT3 hyperphosphorylation through mechanisms other than overdosed cytokines and growth factors.

Head and neck squamous cell carcinoma or, in brief, head and neck cancer ( $\mathrm{HNC}$ ), is the most common epithelial malignancy in the upper aerodigestive tract. STAT3 is activated early during HNC carcinogenesis. ${ }^{21,22}$ The constitutive activation of STAT3 in HNC promotes cell proliferation, cell survival, angiogenesis, immune evasion, epithelial-mesenchymal transition and chemoresistance. ${ }^{9,23-25}$ The inhibition of STAT3 leads to growth inhibition and increased apoptosis in HNC. ${ }^{21,26-28}$ Therefore, STAT3 may represent a candidate oncogene to which HNC is addicted. The overactivation of STAT3 in HNC is induced by epithelial growth factors ${ }^{21,29,30}$ and the cytokine IL- $6^{31-33}$ that are excessively secreted by cancer cells via autocrine and paracrine mechanisms.

Tobacco has a critical role in the etiology of HNC. ${ }^{34-36}$ Tobacco chewing correlates with STAT3 activation in early oral carcinogenesis, ${ }^{22}$ and smokeless tobacco compounds can activate STAT3 in HNC cell lines. ${ }^{37}$ Moreover, cigarette smoke exposure increases phosphorylation of STAT3 in several types of cancerous and non-cancerous epithelial cells. ${ }^{38,39}$ Although they are considered to be field carcinogens, how tobacco extracts activate STAT3 in HNC is unclear. Notably, tobacco carcinogens are also strong inducers of reactive oxygen species (ROS) in cells. ${ }^{40-43}$ ROS can induce or enhance STAT3 activation through various mechanisms. ${ }^{44-46}$ Therefore, we hypothesized that in HNC, tobacco extracts may contribute to the constitutive activation of STAT3 in an ROS-dependent manner.

We have previously found that a sentrin/SUMO2/3-specific protease, SENP3, is a redox-sensitive molecule that accumulates under cellular oxidative stress. ${ }^{47,48}$ Increased SENP3 regulates the functions of its substrates in cancer cells through the removal of SUMO2/3 modification, leading to enhanced cell proliferation, tumorigenesis, angiogenesis and epithelial-mesenchymal transition. ${ }^{47-50}$ Many SENP3 substrates are transcription factors

\footnotetext{
${ }^{1}$ Shanghai Key Laboratory of Tumor Microenvironment and Inflammation, Department of Biochemistry and Molecular Cell Biology, Institutes of Medical Sciences, Shanghai Jiao

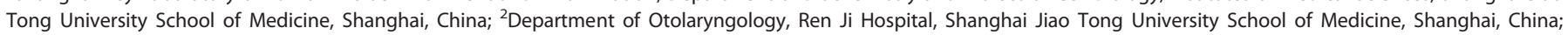

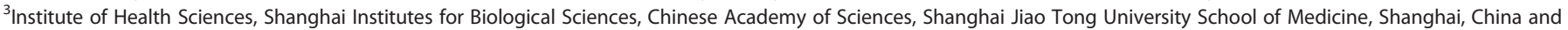

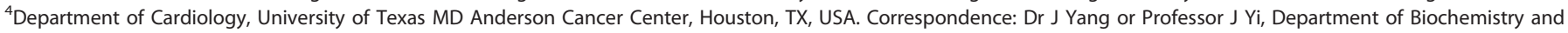
Molecular Cell Biology, Institutes of Medical Sciences, Shanghai Jiao Tong University School of Medicine, 280 S. Chongqing Road, Shanghai 200025, China.

E-mail: yangjieyj@shsmu.edu.cn or yijing@shsmu.edu.cn

Received 7 August 2015; revised 1 March 2016; accepted 11 March 2016; published online 16 May 2016
} 
or co-factors. ${ }^{47,50}$ Our preliminary data have implicated a potential interaction between SENP3 and STAT3. The present study attempted to determine whether SENP3 contributes to the constitutive activation of STAT3 and the development of HNC, specifically when stimulated by tobacco extract.

4-(Methylnitrosamino)-1-(3-pyridyl)-1-butanone (NNK) is one of the major components of cigarette smoke and potent carcinogen. ${ }^{51}$ In the present study, we demonstrated that the exposure of several HNC cell lines to NNK induced the rapid phosphorylation of STAT3 at the tyrosine 705 (Y705), a rapid generation of ROS, accompanied by an ROS-dependent increase in SENP3. SENP3 could enhance STAT3 phosphorylation through de-conjugating SUMO2/3 modification at the lysine 451 site of STAT3. SUMOylation of this site promoted the binding of STAT3 with its phosphatase in the nucleus, TC45. Thus, SENP3mediated STAT3 deSUMOylation led to an enhancement of phosphorylation induced by both NNK and IL-6. We also demonstrated a significant correlation between SENP3 protein levels and STAT3 Y705 phosphorylation levels in human laryngeal carcinoma specimens. These findings provide a novel mechanistic explanation for the hyperphosphorylation of STAT3 in HNC epithelia.

\section{RESULTS}

SENP3 protein level correlates with smoking and STAT3 activation in laryngeal carcinoma

To assess whether SENP3 involves in HNC development, we performed immunohistochemistry in human laryngeal carcinoma and other laryngeal specimens. The results showed that the expression of SENP3 was strongly positive in the nuclei of the epithelial cells in the majority of laryngeal carcinoma tissues. The positive scores of SENP3 (positive stain areas) displayed a gradual increase from vocal polyps to paracarcinoma tissues to laryngeal dysplasias to laryngeal carcinomas. The level of SENP3 protein was markedly higher in cancerous tissues than in other tissues (Figures $1 a$ and b). Classifying the laryngeal carcinoma specimens based on the cigarette smoking history of patients, we found higher SENP3-positive scores in the specimens derived from smoking patients (Figures 1c and d).

STAT3 phosphorylation at tyrosine 705 is required for its dimerization and nuclear translocation, thus serving as a typical marker for cytokine/growth factor-induced STAT3 activation. ${ }^{1,52,53}$ Next, we evaluated whether the expression levels of SENP3 correlated with Y705 phosphorylated STAT3 (pY705-STAT3) in 159 laryngeal carcinomas using serial sections. Positive immunohistochemical staining for pY705-STAT3 was visible in the nuclei in the majority of cancerous epithelial cells. In parallel, SENP3 staining was markedly positive in the same areas of the same samples. Some tissues displayed weaker pY705-STAT3 staining, which corresponded to weaker SENP3 staining in the same areas (Figure 1e). The analysis of these paired sections demonstrated a linear correlation between the positive scores of both proteins in each specimen (Figure 1f, left). In addition, the positive intensity of both proteins in the same areas within one specimen also displayed a linear correlation (Figure 1f, right).

NNK induces STAT3 phosphorylation and increase of SENP3 protein levels in HNC cells in an ROS-dependent manner

To determine whether tobacco extracts induce STAT3 activation in HNC, the Hep-2 laryngeal carcinoma cell line was exposed to $1 \mu \mathrm{m}$ of NNK over a short time course. STAT3 activation was analyzed by immunoblotting (IB) for pY705-STAT3 (in brief, p-STAT3). A rapid increase in P-STAT3 starting at $5 \mathrm{~min}$ and lasting for $1 \mathrm{~h}$ was observed with no change in the total amount of STAT3 protein. This activation pattern was comparable to but more rapid and transient than that induced by IL- 6
(Figure 2a, left). We then examined the effect of NNK in two other HNC cell lines, KB, which was derived from an epidermal carcinoma of the mouth, and HN30, which was derived from an epidermal carcinoma of pharynx. A similar activation was found in these HNC cells (Figure 2a, right). To further investigate whether NNK-induced STAT3 Y705 phosphorylation is tissue specific, we examined p-STAT3 in additional cell lines derived from carcinomas of the lung, brain, stomach, liver, breast and colon. The results showed that the HNC cell line Hep-2 and the gastric carcinoma cell line MGC803 had higher basal p-STAT3 levels and the robustly increased p-STAT3 at the first $5 \mathrm{~min}$, while in A549, a lung cancer cell line, the phosphorylation of STAT3 was much later (Supplementary Figure S1), indicating that STAT3 can be preferentially activated by NNK in HNC cells.

We next evaluated whether the effect of NNK depended on the canonical IL-6/receptor/JAK pathway. Cytokine deprivation and JAK inhibition assays showed that NNK activating STAT3 required the presence of basal levels of IL- 6 and JAK, because the anti-IL- 6 antibody and JAK inhibitor prevented pY705 phosphorylation in response to NNK treatment (Figure $2 \mathrm{~b}$ ). These data suggested that NNK might potentiate IL-6-induced STAT3 activation. We then exposed Hep-2 cells to much lower doses of IL-6 or NNK. While these two reagents could both induce STAT3 activation at low doses, the combination of IL- 6 and NNK led to a markedly stronger activation than that of IL-6 alone (Figure 2c).

Cigarette smoking induces oxidative stress ${ }^{41,54}$ and SENP3 is a SUMO2/3 protease responsive to oxidative stress. ${ }^{47}$ Based on these notions and the aforementioned correlation between SENP3 levels and smoking in laryngeal squamous cell carcinoma specimens, we speculated that NNK could induce an increase in SENP3 and simultaneously an increase in ROS generation in HNC cell lines. A measurement of the relative levels of ROS indeed showed a time-dependent increase in ROS generation after NNK treatment (Figure $2 \mathrm{~d}$ ). The results of immunoblots demonstrated an SENP3 induction in parallel to p-STAT3 induction in Hep-2 cells exposed to NNK (Figure 2e, left). Our previous studies showed that the rapid increase in SENP3 protein level was resulted from an accumulation of protein due to cysteine oxidation, which could be blocked by antioxidants. ${ }^{47}$ Cells were then pre-incubated with the anti-oxidant $\mathrm{N}$-acetyl-Lcysteine (NAC) before NNK treatment. This pre-treatment abolished NNK-induced SENP3 accumulation to a great extent. Interestingly, p-STAT3 induction by NNK was also blocked by pre-treatment with NAC (Figure 2e, left). Consistently, NNKinduced increases in both SENP3 and p-STAT3 were detectable in two other $\mathrm{HNC}$ cell lines, $\mathrm{KB}$ and $\mathrm{NH} 30$, and NAC prevented these increases (Figure 2e, right).

These results indicate that NNK can activate STAT3 in HNC cells, which enhances the pre-existing IL-6/JAK/STAT3 signaling pathway. NNK simultaneously induces an increase of SENP3 in an ROSdependent manner. We next sought to investigate the role of SENP3 in NNK- and IL-6-induced STAT3 activation.

SENP3 enhances the basal and NNK- or IL-6-induced STAT3 phosphorylation in HNC cells

After stably knocking down SENP3 expression using shRNA in Hep-2 cells, we examined p-STAT3 levels in these cells exposed to NNK over a short time course. The results of immunoblots showed that the basal and NNK-induced levels of p-STAT3 were significantly suppressed in SENP3 knocked-down (sh-SENP3) cells compared with non-specific siRNA control (sh-NC) cells (Figure 3a). Consistently, in two other HNC cell lines, transiently knocking-down SENP3 impaired the basal and NNK-induced p-STAT3 levels (Figure $3 b$ ). Moreover, the increase in p-STAT3 levels induced by IL-6 was similarly suppressed in SENP3-stably knockdown (sh-SENP3) cells. Notably, overexpression of SENP3 


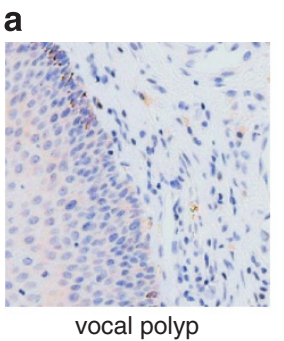

b $\quad \mathrm{N}=12 \quad 52 \quad 10 \quad 52$

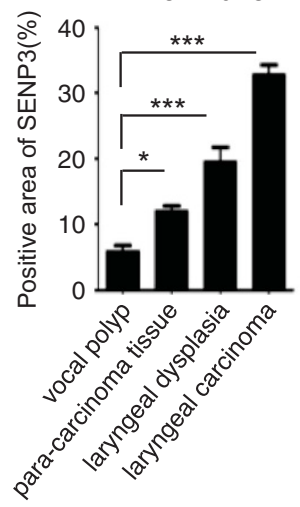

e SENP3

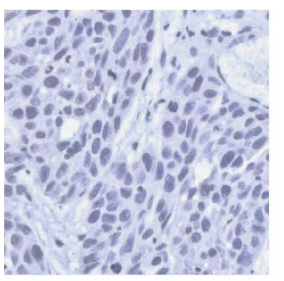

laryngeal carcinoma 1

f

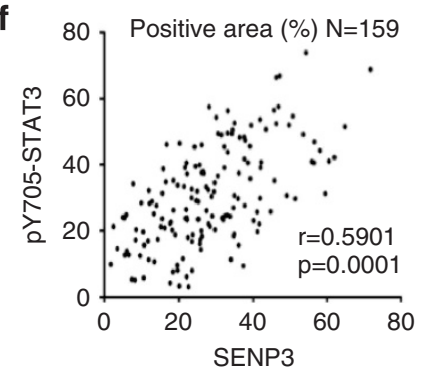

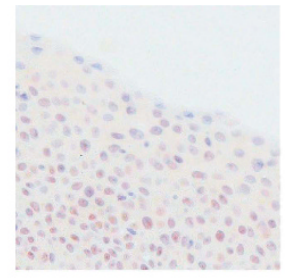

laryngeal dysplasia

C non-smoking

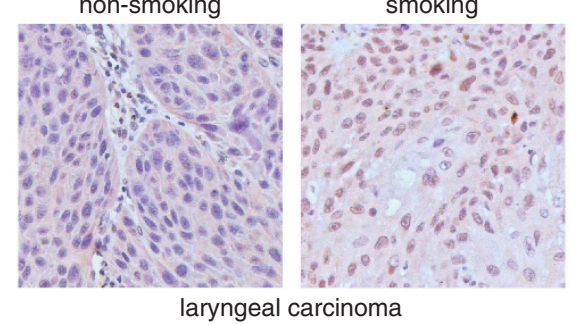

laryngeal carcinoma

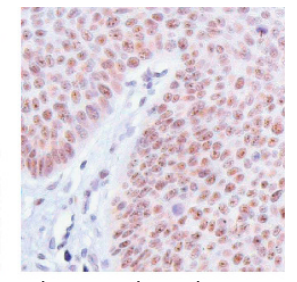

laryngeal carcinoma

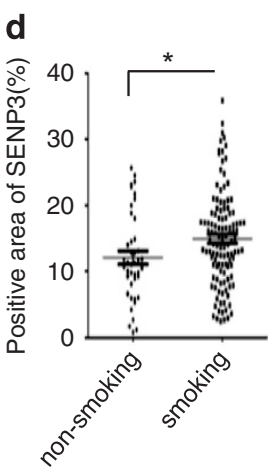

PY705-STAT3
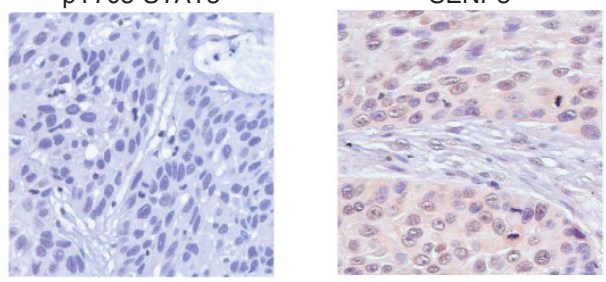

laryngeal carcinoma 2

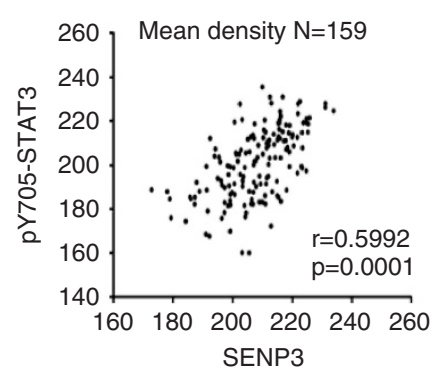

Figure 1. SENP3 protein level correlates with smoking and STAT3 activation in laryngeal carcinoma. (a) Immunohistochemistry for SENP3 was performed in sections derived from vocal polyp $(n=12)$, laryngeal dysplasia $(n=10)$, laryngeal carcinoma specimens and their corresponding para-carcinoma tissues $(n=52)$. The representative images were shown. (b) The relative positive stain areas of SENP3 in each specimen were measured, and the average percentage of each group was displayed in bar chart. All data were shown with the means \pm s.d. of all specimen in different groups. ${ }^{*} P<0.05,{ }^{* *} P<0.001$. (c) Immunohistochemistry for SENP3 was performed in 159 laryngeal carcinoma specimens classified based on cigarette smoking $(n=117)$ and non-smoking $(n=42)$ patients. The representative images were shown. (d) The relative positive stain areas of SENP3 in two groups were measured and were displayed in scatterplot. ${ }^{*} P<0.05$. (e) Immunohistochemistry for SENP3 and pY705STAT3 was performed in serial sections derived from specimens of different laryngeal cancers. Two representative specimens were shown. (f) The positive scores of protein levels of SENP3 and pY705-STAT3 based on the data in (e) were shown in two indexes: the percentages of positive stain areas of the both proteins in each specimen (left) and the average intensities of positive stain of the both proteins in the same areas within each specimen (right). $r=$ the coefficients of correlation.

could enhance p-STAT3 levels under basal conditions but was unable to further increase the IL-6-induced increase in p-STAT3 levels (Figure 3c).

These data indicate that the presence of SENP3 is important for the basal and NNK- or IL-6-induced STAT3 phosphorylation in HNC cells.
SENP3 enhances STAT3 phosphorylation by suppressing the interaction between STAT3 and its phosphatase TC45 in the nucleus The enhancement of STAT3 phosphorylation can be attributed to increased phosphorylation in the cytoplasm or nucleus. ${ }^{2}$ SENP3 is preferentially localized in the nucleoli. ${ }^{55}$ Our previous studies demonstrated that SENP3 accumulates in the 
a

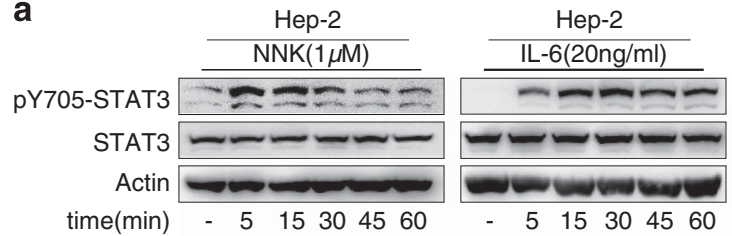

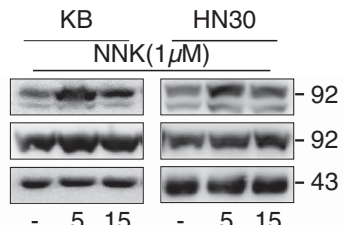

$\mathrm{NNK}(1 \mu \mathrm{M})$

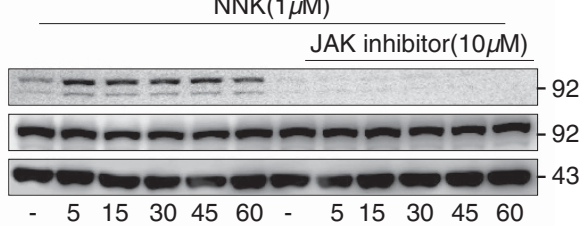

d

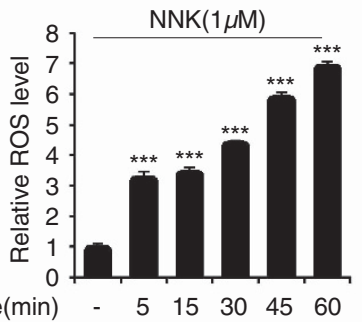

e

Hep-2 NNK $(1 \mu \mathrm{M})$ NAC(antioxidant)

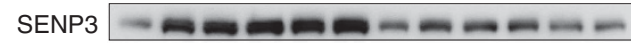

pY705-STAT3

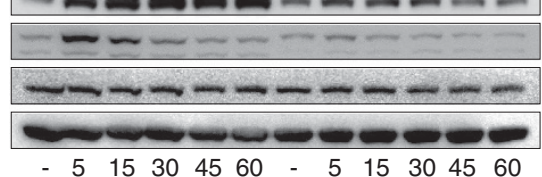

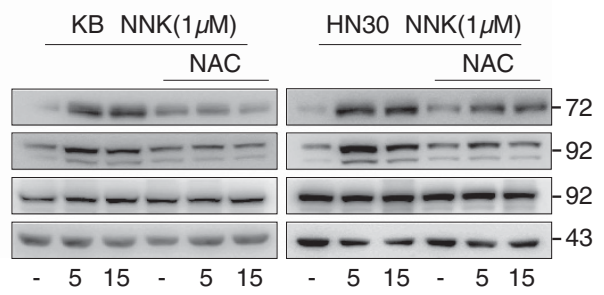

Figure 2. NNK induces STAT3 phosphorylation and increase of SENP3 protein levels in HNC cells in an ROS-dependent manner. (a) The levels of STAT3 phosphorylation at tyrosine 705 (in brief, pY705-STAT3) were determined by IB in Hep-2 cells exposed to $1 \mu \mathrm{m}$ NNK and $20 \mathrm{ng} / \mathrm{ml}$ IL-6 respectively for the indicated time (left), and in KB or HN30 cells exposed to $1 \mu \mathrm{m}$ NNK for indicated time (right). (b) The pY705-STAT3 levels were determined by IB in Hep-2 cells exposed to $1 \mu \mathrm{m}$ NNK in the presence or absence of $1 \mu \mathrm{g} / \mathrm{ml}$ neutralized antibody against IL-6 (left) and $10 \mu \mathrm{M}$ JAK inhibitor (right) for the indicated time. Anti-IL-6 antibody and JAK inhibitor were pre-administered for $1 \mathrm{~h}$. (c) The pY705-STAT3 levels were determined by IB in Hep- 2 cells exposed to lower doses of NNK $(0.01 \mu \mathrm{M})$ or IL- $6(2 \mathrm{ng} / \mathrm{ml})$ alone or in combination for the indicated time. (d) ROS level was determined by DCFH-DA staining and flow-cytometric analysis in Hep2 cells exposed to $1 \mu \mathrm{m}$ NNK for the indicated time. Error bars represent \pm s.d. for triplicate experiments. ${ }^{* * *} P<0.001$. (e) The SENP3 and pY705-STAT3 levels were determined by IB in Hep-2, $\mathrm{KB}$ and HN30 cells exposed to $1 \mu \mathrm{M}$ NNK in the presence or absence of $5 \mathrm{~mm}$ NAC for the indicated time.

nucleoplasm under conditions of oxidative stress, where it interacts with nuclear proteins, ${ }^{47}$ especially transcription factors. ${ }^{47,50}$ To clarify the regulatory role of SENP3 in STAT3 activation, we used SENP3-overexpressing and -knocking-down Hep-2 cells (Supplementary Figure S2) to examine the levels of p-STAT3 in the nuclear and cytoplasmic fractions, respectively, and found that changes related to the levels of SENP3 occurred in the nucleus rather than in the cytoplasm (Figure 4a). The overexpression of SENP3 could promote basal and NNKinduced p-STAT3 levels in the nucleus, although it could not further increase the IL-6-induced p-STAT3 levels in the nucleus. Knocking-down of SENP3 markedly decreased the basal and NNK- or IL-6-induced p-STAT3 levels in the nucleus (Figure 4a).

The enhancement of STAT3 phosphorylation may be caused not only by increased phosphorylation, but also by decreased dephosphorylation. ${ }^{56}$ We next sought to determine the side on which SENP3 might act. The nuclear phosphatase TC45 can mediate STAT3 dephosphorylation in the nucleus. ${ }^{57,58}$ Transfection of Hep-2 cells with increasing doses of Flag-TC45 revealed that TC45 could suppress p-STAT3 levels in a dose- dependent manner, regardless of the induction by NNK or IL-6 (Figure 4b), suggesting that the phosphatase activity of TC45 is essential for the dephosphorylation of STAT3 in Hep-2 cells. To examine whether SENP3 had any effect on the negative regulation of STAT3 phosphorylation by TC45, we assessed changes in p-STAT3 levels in SENP3-stablyknockdown Hep-2 cells (Hep-2-sh-SENP3) treated with the phosphatase inhibitor sodium orthovanandate $\left(\mathrm{Na}_{3} \mathrm{VO}_{4}\right)$. The p-STAT3 levels in Hep-2-sh-SENP3 and control (Hep-2-shcontrol) cells exposed to NNK plus $\mathrm{Na}_{3} \mathrm{VO}_{4}$ were compared. The results showed that the levels of p-STAT3 were markedly enhanced in the presence of $\mathrm{Na}_{3} \mathrm{VO}_{4}$, but this enhancement in p-STAT3 returned to basal levels upon SENP3 knockdown (Supplementary Figure S3). These results implied that SENP3 might enhance STAT3 phosphorylation through its effects on the phosphatase TC45.

SUMOylation and deSUMOylation modulate the interactions between proteins. Because TC45 was reported to interact with STAT3 in the nucleus, ${ }^{57,58}$ we examined whether SENP3 altered the association between TC45 and STAT3. Co-immunoprecipitation (co-IP) analysis revealed that 
a

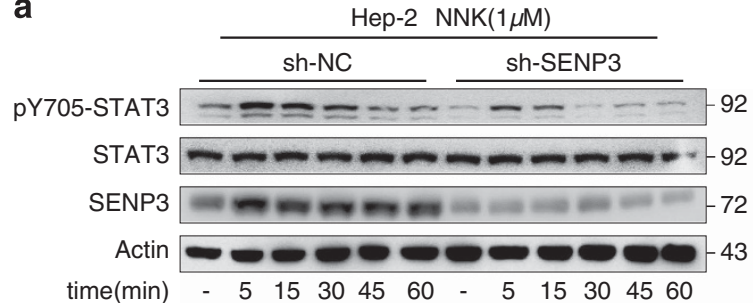

b

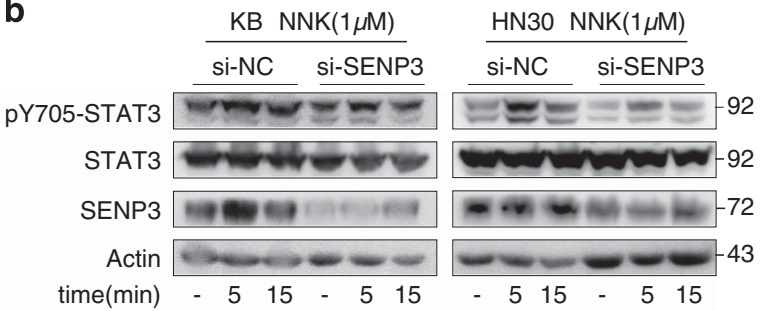

C

Hep-2 IL-6(20ng/ml)
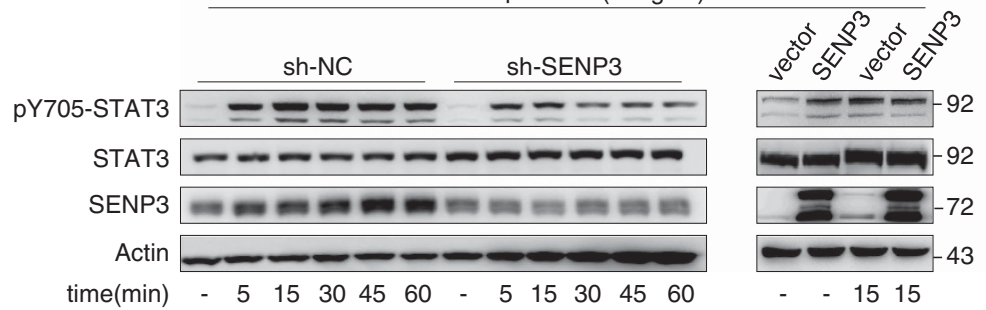

Figure 3. SENP3 enhances the basal and NNK-or IL-6-induced STAT3 phosphorylation in HNC cells. (a-c) The pY705-STAT3 levels were determined by IB in the cells with various gene interferences and treatments. (a) Hep-2 cells stably expressing non-specific shRNA (sh-NC) or SENP3 shRNA (sh-SENP3) were exposed to $1 \mu \mathrm{m}$ NNK for the indicated time. (b) KB and HN30 cells with SENP3 knockdown (si-SENP3) for $48 \mathrm{~h}$ were exposed to $1 \mu \mathrm{m}$ NNK for the indicated time. (c) Hep-2 cells with either sh-SENP3 (left) or overexpressed SENP3 (right) were exposed to $20 \mathrm{ng} / \mathrm{ml} \mathrm{IL-6}$ for the indicated time.

a

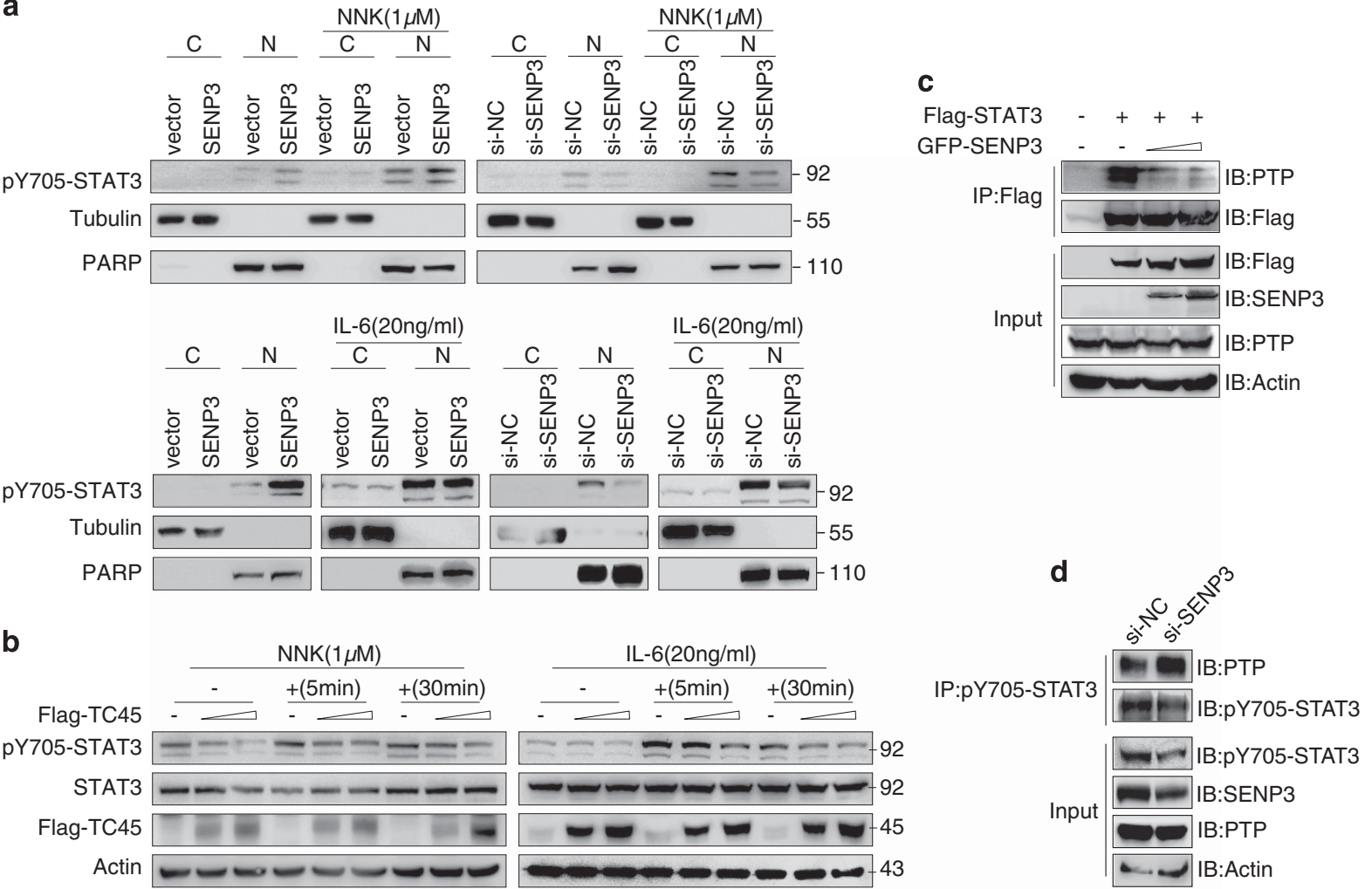

Figure 4. SENP3 enhances STAT3 phosphorylation by suppressing the interaction between STAT3 and its phosphatase TC45 in the nucleus. (a) The pY705-STAT3 levels were examined in the fractions of cytoplasm and nucleus, respectively. Hep-2 cells with SENP3 overexpression or si-SENP3 for $48 \mathrm{~h}$ were exposed to $20 \mathrm{ng} / \mathrm{ml} \mathrm{IL}-6$ for $15 \mathrm{~min}$ or $1 \mu \mathrm{m}$ NNK for $30 \mathrm{~min}$. Tubulin and PARP were taken as the internal controls for cytoplasm ' $C$ ' and nucleus ' $N$ ', respectively. (b) The pY705-STAT3 levels were examined in Hep-2 cells. Hep-2 cells were transfected with the gradient concentrations of Flag-TC45 for $24 \mathrm{~h}$ and then exposed to $1 \mu \mathrm{m}$ NNK and $20 \mathrm{ng} / \mathrm{ml} \mathrm{IL-6}$, respectively, for the indicated time. (c) The interaction of STAT3 with TC45 was detected by co-IP. HEK293 T (293T) cells were transfected with the gradient concentrations of GFP-SENP3 and Flag-STAT3 for $48 \mathrm{~h}$. Immunoprecipitation of Flag-tagged STAT3 was performed to detect TC45 using anti-PTP antibody. (d) The interaction of endogenous TC45 with pY705-STAT3 was determined by co-IP in the lysates of Hep-2 cells with si-SENP3 for 48 h. IP was performed using anti-pY705-STAT3 antibody. 
endogenous TC45 formed a complex with Flag-tagged STAT3 (TC45 was blotted by the antibody against PTP). Indeed, the interaction between Flag-STAT3 and endogenous TC45 was weakened by overexpression of increasing SENP3 (Figure 4c). Furthermore, the interaction between endogenous TC45 and endogenous phosphorylated STAT3 was augmented a

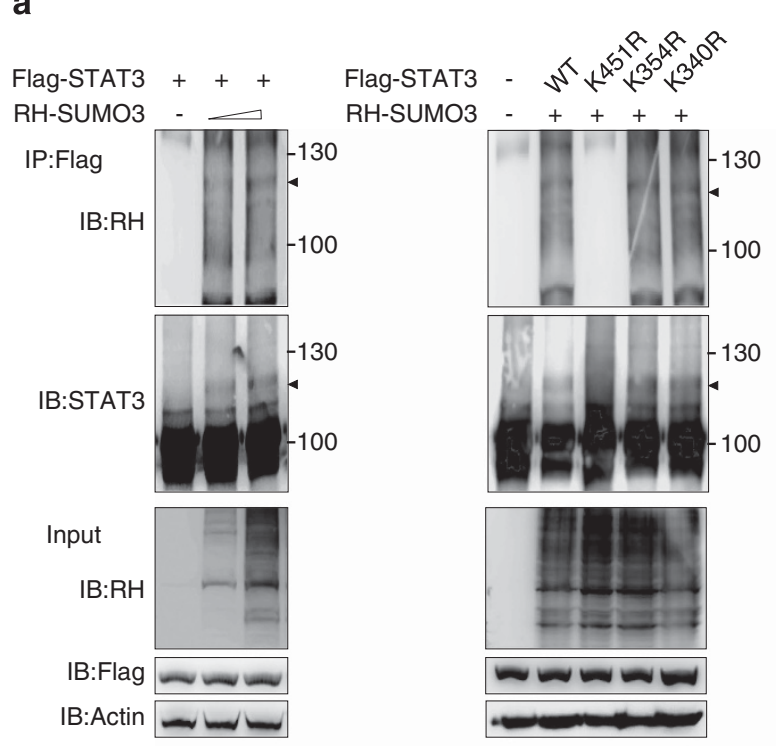

C

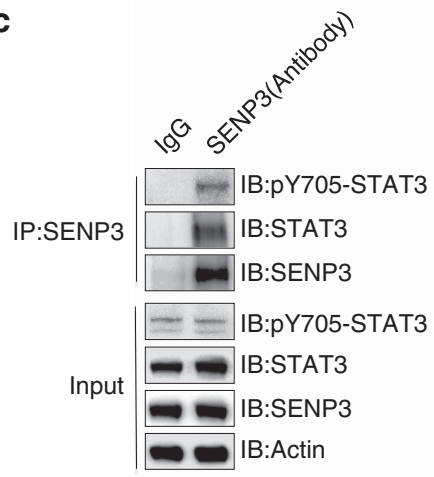

d

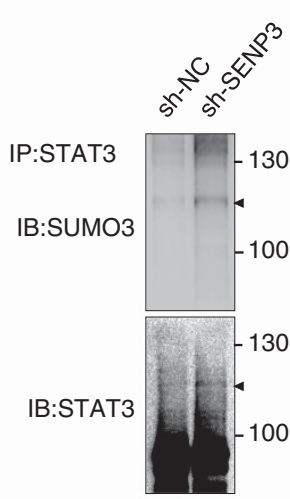

Input

IB:SUMO3

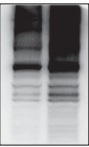

IB:STAT3

IB:SENP3

IB:Actin
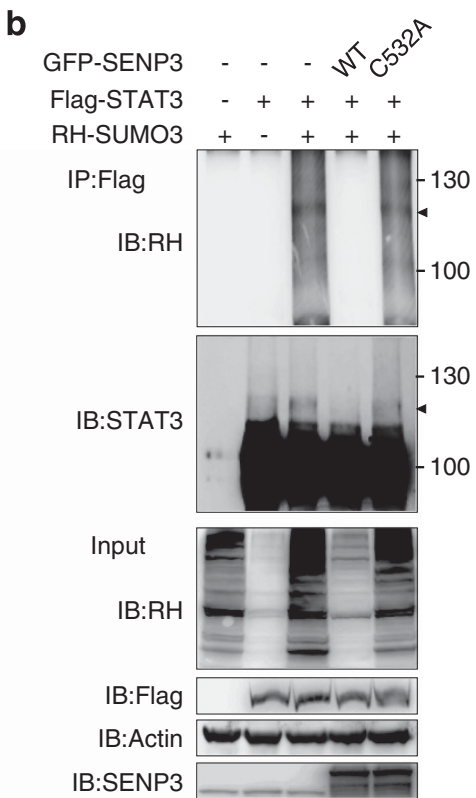

e
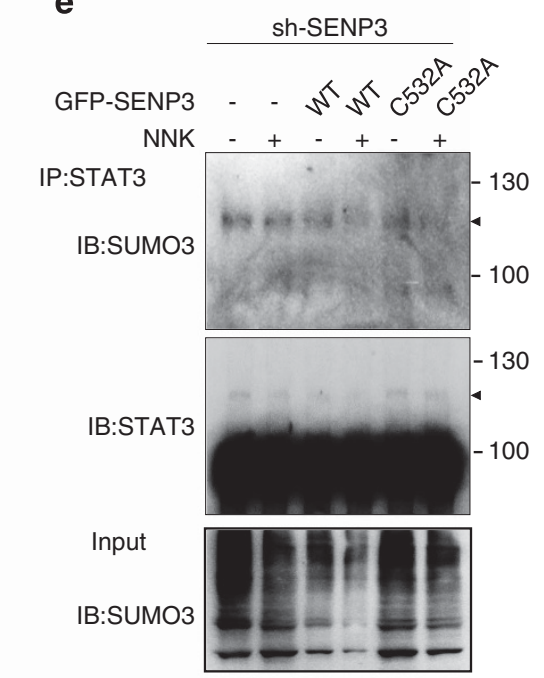

IB:pY705-STAT3

IB:SENP3 -1 -

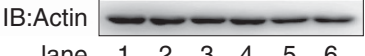

Figure 5. SENP3 de-conjugates SUMO2/3 at lysine 451 of STAT3 to halt its association with TC45. (a, b) SUMO3 conjugates of STAT3 were determined by co-IP in HEK293T cells. Cells were transfected with FLAG-STAT3 and RH-SUMO3 for 48 h. Co-IP was performed using Flag-M2 beads for immunoprecipitation and using anti-RH and anti-STAT3 antibodies for IB. (a) Cells were transfected with RH-SUMO3 in gradient concentrations (left). Cells were co-transfected with Flag-tagged WT or mutated STAT3 (K451R, K354R and K340R) and RH-SUMO3 (right). (b) Cells were co-transfected with GFP-tagged WT or mutated SENP3 (C532A) and FLAG-STAT3. (c) Endogenous SENP3/pY705-STAT3 or STAT3 interaction was determined by co-IP in Hep-2 cells. (d) Endogenous SUMO3 conjugates of STAT3 were determined by co-IP in the lysates of HEK293T-sh-SENP3 stable cells. (e) Endogenous SUMO3 conjugates of STAT3 were determined by co-IP in the lysates of Hep2-sh-SENP3 stable cells when GFP-tagged WT or mutated SENP3 (C532A) putting back and $1 \mu \mathrm{m}$ NNK administration for 30 min. Arrowheads indicated SUMO3conjugated STAT3 in (a, b, d, e). (f) The pY705-STAT3 levels were determined by IB in the lysates of Hep2-sh-NC and Hep2-sh-SENP3 stable cells when co-transfected with GFP-tagged WT or mutated STAT3 (K451R). (g) The interaction of STAT3 with TC45 was determined by co-IP in the lysates of HEK293T cells transfected with Flag-tagged WT STAT3 or SUMOless mutant STAT3 (K451R) and STAT3-SUMO3 fusion vector. (h) The interaction of STAT3 with TC45 was determined by FRET analysis in Hep-2 cells transfected with Flag-tagged WT STAT3 or STAT3-SUMO3 fusion vector. Bar, $10 \mu \mathrm{m}$. Percentages of N-FRET values in 10 cells were showed. $* * * P<0.001$. (i) The interaction of STAT3 with TC45 was determined by co-IP in the lysates of HEK293T cells transfected with Flag-tagged WT TC45 or SIM mutant TC45 (V109A/L112A). (j) A model illustrating the critical role of SUMOylation in the association of STAT3 with TC45. 
f

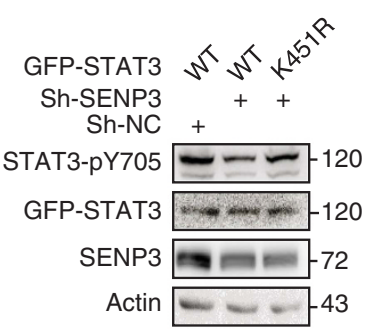

g

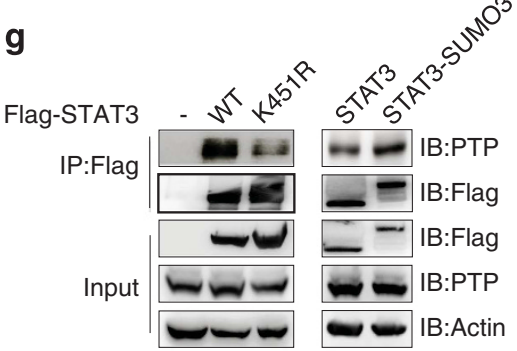

h
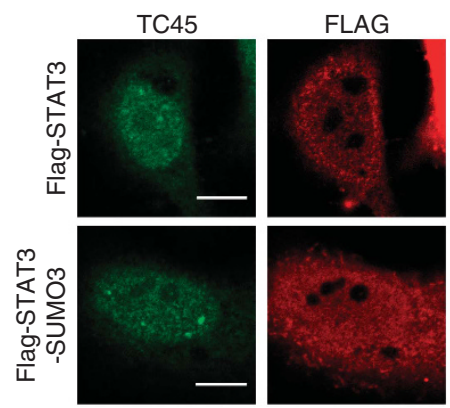

N-FRET

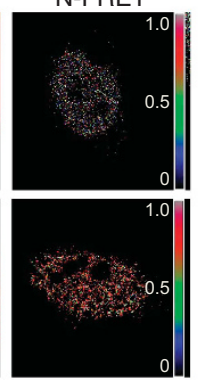

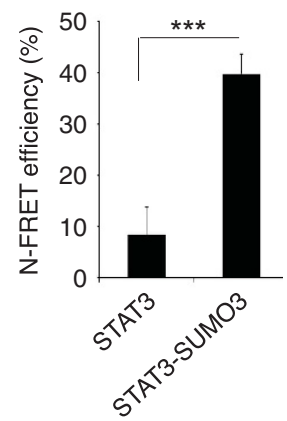

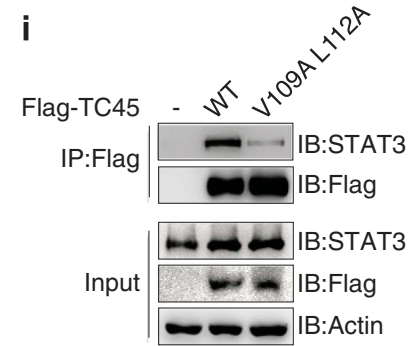

j

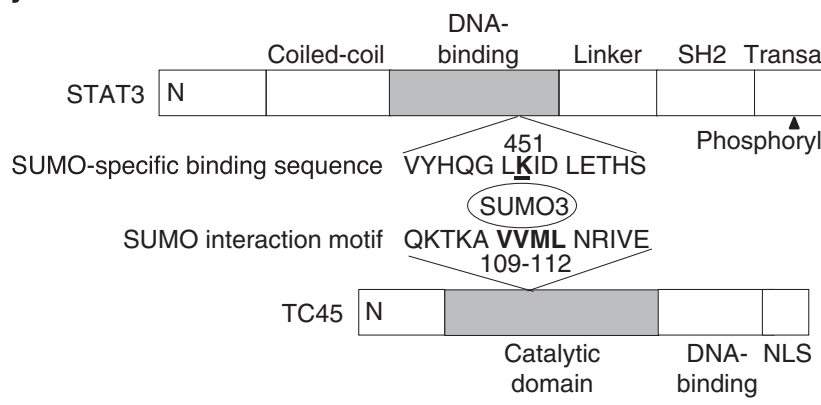

Figure 5. Continued.

by SENP3 knockdown (Figure 4d). These results suggest that SENP3 negatively regulates the association between STAT3 and TC45.

SENP3 de-conjugates SUMO2/3 at lysine 451 of STAT3 to halt its association with TC45

We speculated that the effect of SENP3-mediated enhancement in STAT3 phosphorylation depended on its enzymatic activity to de-conjugate SUMO2/3 from STAT3.

We first tested whether STAT3 had a SUMO2/3 modification in HEK293T cells. The SUMOylation of STAT3 was examined using Flag IP assays. The results of IB showed that SUMO3 conjugation of STAT3 displayed a prominent band on the gel at $120 \mathrm{kDa}$ and was increased with the dose-dependent overexpression of SUMO3 (Figure 5a, left). The probability of SUMOylation site of STAT3 was predicted using open software (Supplementary Figure S4); and K451, K354 and K340 were highly scored. We then constructed plasmids of wild-type (WT) STAT3 or the mutants in which the predicted Lys residues of the SUMOylation sites were replaced by Arg that did not allow SUMOylation. The results of co-IP showed that STAT3 was conjugated by SUMO3 at K451, as the WT STAT3 was pulled-down with $\mathrm{SUMO} 3$ conjugates, displaying the SUMO band, and the K451R mutant lacked this band; however, these bands were retained in the remaining two mutants (Figure $5 \mathrm{a}$, right). The co-IP based on the overexpression of STAT3 and SENP3 showed that SENP3 WT was able to remove SUMO3 from STAT3, whereas the SENP3 mutant (C532A, loss of enzyme activity) was not able to do so (Figure $5 b$ ). The co-IP based on the endogenous setting of Hep-2 cells showed that SENP3 indeed interacted with the total and phosphorylated STAT3 (Figure $5 \mathrm{c}$ ). These data verified STAT3 as the substrate of SENP3. Next, siRNA against SENP3 was used to assess the changes in endogenous SUMOylation of STAT3. Knocking-down of SENP3 dramatically enhanced the basal endogenous SUMOylation conjugates in both HEK293T cells (Figure 5d) and Hep-2 cells (Figure 5e). Moreover, after the WT or mutant C532A SENP3 were put back to SENP3knockdown Hep-2 cells and cells were exposed to NNK, the extents of endogenous SUMO2/3 conjugates of STAT3 were changed: the WT SENP3 removed SUMO, while the mutant could not (Figure 5e). Notably, upon NNK treatment, the deSUMOylation was enhanced (compare lanes 2, 4 and 6 with lanes 1, 3 and 5, respectively, in Figure 5e), which might be due to the remaining endogenous SENP3's effect. Consistently, fewer SUMO2/3 modifications of STAT3 and fewer global SUMO2/3 conjugations well coincided with more phosphorylation of STAT3 in these samples. Moreover, overexpression of SUMOless mutant STAT3 K451R in the SENP3-knockdown cells, which mimicked deSUMOylated status of STAT3, indeed caused an increase in STAT3 phosphorylation (compared with overexpression of WT STAT3, Figure 5f). To exclude that the K451R mutant might lose lysine modifications other than SUMOylation and thus mediate off-target effects, we examined ubiquitination and acetylation of this mutant. The results of co-IP showed that two types of modifications had similar 


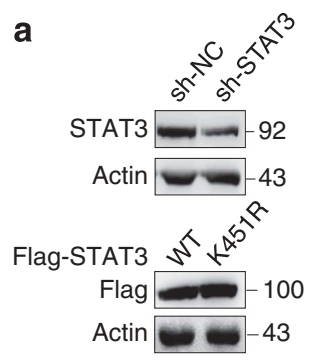

b

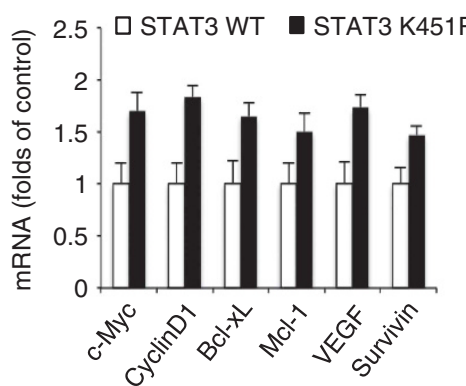

C

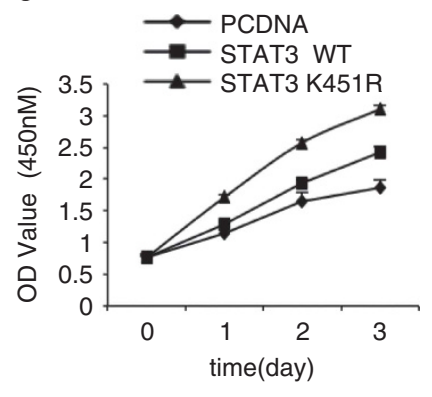

d

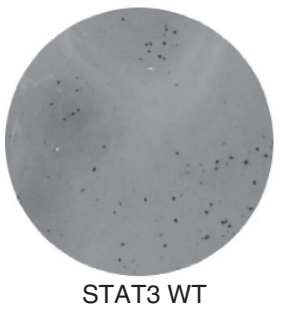

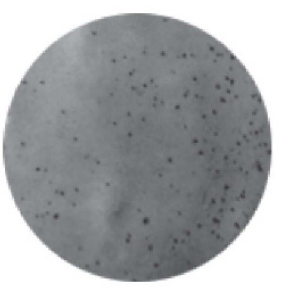

STAT3 K451R

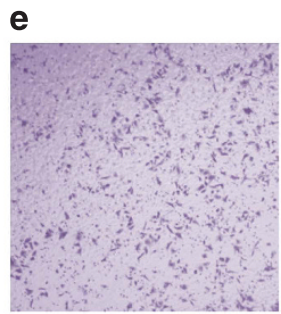

STAT3 WT

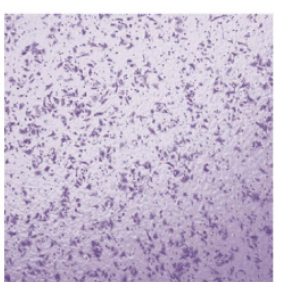

STAT3 K451R
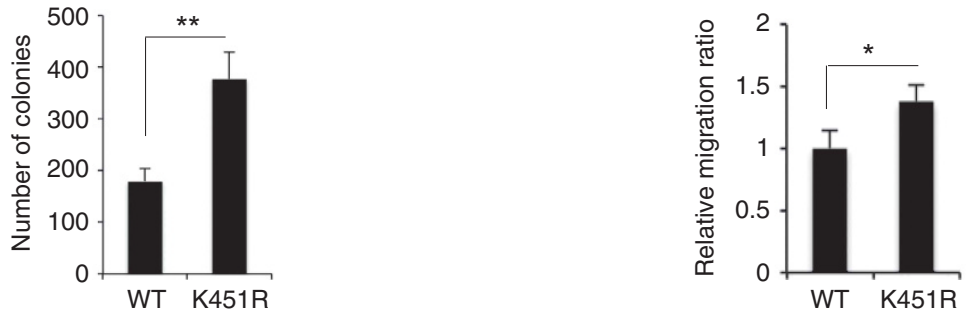

Figure 6. DeSUMOylation of STAT3 enhances its transcriptional activity and oncogenic function in HNC cells. (a-e) Hep2-sh-STAT3 stable cells were added back with Flag-tagged WT STAT3 or SUMOless mutant STAT3 (K451R) before undergoing various assays. (a) The efficiency of ShRNA and overexpression of SENP3 was determined by IB. (b) The mRNA levels of STAT3 target genes C-Myc, Cyclin D1, BCl-xL, Mcl-1, VEGF and Survivin were determined by qRT-PCR at $48 \mathrm{~h}$ post transfection. (c) The viable cell numbers were measured by CCK 8 at $0,24,48$ and $72 \mathrm{~h}$ post transfection. (d) The numbers of colonies in soft agar were measured. ${ }^{*} P<0.01$. (e) The cell migration ability was analyzed by Transwell migration assay. ${ }^{*} P<0.05$. (b, d, e) Error bars represent \pm s.d. for triplicate experiments. (c) Error bars represent \pm s.d. for three replication wells. The experiment repeated three times.

extents between the WT and mutant (Supplementary Figure S5), which justified the usage of K451R.

These data proposed that SENP3 halts the interaction of TC45 and p-STAT3 via deSUMOylating STAT3. To clarify whether the SUMO2/3 modification of STAT3 promoted its association with TC45, we examined the binding between endogenous TC45 and Flag-STAT3 WT or K451R. We further constructed a fusion protein with a SUMO3 linked to the C-terminus of STAT3. As expected, the interaction was greatly weakened between TC45 and SUMOless STAT3, but in contrast, markedly augmented between TC45 and SUMO-fused STAT3 (Figure $5 \mathrm{~g}$ ). Alternatively, these interactions were evaluated by fluorescence resonance energy transfer (FRET) assay. The intensity of interaction in the nuclei was obviously higher in cells with SUMO-fused STAT3 (Figure 5h). These assays provided direct evidence for that SUMOylated STAT3 facilitates the recruitment of TC45.

The SUMO-interacting motif (SIM) V/I-X-V/I-V/I that was identified to bind with SUMO (1-3) or SUMOylated proteins exists in nearly all proteins known to be involved in SUMOdependent processes. ${ }^{59}$ We predicted a highly scored SIM in TC45 at 109-112 (VVML) using open software (Supplementary Figure S6). To further address whether an SIM in TC45 mediated its binding with SUMOylated STAT3, we constructed a TC45 SIM mutant (V109A, L112A) in which the predicted essential amino-acid residues of SIM, Val 109 and Leu 112 were replaced by Ala that did not allow binding with SUMO or SUMOylated proteins. The co-IP results showed that the SIM-mutated TC45 was almost incapable of binding with STAT3 (Figure 5i), demonstrating that SUMOylation of STAT3 is critically required for its association with TC45 via SIM (Figure 5j) and is important for the inhibition of STAT3 activity in HNC cells. These data explained the role of SENP3 in the enhancement of STAT3 phosphorylation.

DeSUMOylation of STAT3 enhances its transcriptional activity and oncogenic function in HNC cells

In an attempt to investigate the biological effects of deSUMOylated STAT3 in Hep-2 cells, we knocked down endogenous STAT3 and simultaneously added back the WT STAT3 or SUMOless mutant K451R, respectively (Figure 6a). These cells were then cultured and analyzed for STAT3 target gene expression, proliferation, colonigenesis and migration. The results of qRT-PCR showed that the most widely recognized target genes that were transcriptionally controlled by STAT3, c-Myc, cyclin D1, Bcl-XL, Mcl-1, VEGF and Survivin were upregulated in K451R-expressing cells (Figure 6b). The capabilities of cell proliferation, colonigenesis in soft agar and cell migration in Transwells were greater in K451R-expressing 
cells (Figures $6 c-e$ ). These data indicated that a deSUMOylated STAT3 is more potent in its transcriptional activity and oncogenic function, which is consistent with the effects of SENP3 in HNC cells and laryngeal carcinoma specimens.

SENP3 has a role in the overactivation of STAT3 and poorer clinicopathological behaviors in HNC

We statistically analyzed the correlation between immunohistochemistry-determined SENP3 protein levels and clinicopathological parameters of 159 laryngeal carcinoma patients. The results demonstrated that higher levels of SENP3 protein were significantly linked to the smoking history, lymph-node metastasis, tumor staging and clinical staging, but were not relevant to gender, degree of cell differentiation or tumor site (Table 1). Finally, we further evaluated the relationships of both p-STAT3 levels and SENP3 levels to these associated clinicopathological parameters. A significant relevance of p-STAT3 levels with SENP3 levels existed in tobacco smoker patients, but p-STAT3 levels and SENP3 levels were much less relevant to each other in non-smoker counterpart. The relevance was more significant in N1-2 nodal metastasis compared with N0 state. The relevance was also more significant

Table 1. Correlation between the clinicopathological features and SENP3 expression

\begin{tabular}{|c|c|c|c|c|}
\hline Characteristics & $\begin{array}{c}\text { Case } \\
\text { no. }\end{array}$ & $\begin{array}{l}\text { Positivity area of } \\
\text { SENP3 (\%) }\end{array}$ & $\begin{array}{c}\text { Non-parametric } \\
\text { test value }\end{array}$ & P-value \\
\hline \multicolumn{5}{|l|}{ Gender } \\
\hline Male & 152 & $28.24 \pm 14.29$ & $Z=-0.604$ & 0.546 \\
\hline Female & 7 & $30.05 \pm 15.64$ & & \\
\hline \multicolumn{5}{|c|}{ Tobacco smoking* } \\
\hline Yes & 117 & $29.81 \pm 14.41$ & $Z=-2.237$ & 0.025 \\
\hline No & 42 & $24.15 \pm 13.29$ & & \\
\hline \multicolumn{5}{|l|}{ Alcohol } \\
\hline Yes & 25 & $33.35 \pm 14.87$ & $Z=-1.874$ & 0.061 \\
\hline No & 134 & $27.38 \pm 14.05$ & & \\
\hline \multicolumn{5}{|l|}{ Nodal status** } \\
\hline No & 111 & $26.37 \pm 14.15$ & $Z=-2.735$ & 0.006 \\
\hline N1-2 & 48 & $32.83 \pm 13.74$ & & \\
\hline \multicolumn{5}{|c|}{ Pathological differentiation grade } \\
\hline Well & 67 & $26.37 \pm 14.15$ & $X^{2}=2.626$ d.f. $=2$ & 0.269 \\
\hline Moderate & 82 & $32.83 \pm 13.74$ & & \\
\hline Poor & 10 & $26.37 \pm 14.15$ & & \\
\hline \multicolumn{5}{|c|}{ Clinical stage (TNM classification)** } \\
\hline I & 52 & $24.56 \pm 13.53$ & $X^{2}=15.789$ d.f. $=3$ & 0.001 \\
\hline II & 39 & $23.86 \pm 9.375$ & & \\
\hline III & 43 & $32.07 \pm 15.74$ & & \\
\hline IV & 25 & $36.64 \pm 14.96$ & & \\
\hline \multicolumn{5}{|l|}{ Tumor stage* } \\
\hline T1 & 52 & $24.56 \pm 13.53$ & $x^{2}=10.407$ d.f. $=3$ & 0.015 \\
\hline T2 & 52 & $26.64 \pm 11.74$ & & \\
\hline T3 & 37 & $31.41 \pm 15.42$ & & \\
\hline T4 & 18 & $37.66 \pm 16.40$ & & \\
\hline \multicolumn{5}{|l|}{ Tumor site } \\
\hline Supraglottic & 50 & $29.28 \pm 11.68$ & $X^{2}=0.452$ d.f. $=2$ & 0.798 \\
\hline Glottic & 91 & $28.44 \pm 16.52$ & & \\
\hline Subglottic & 18 & $27.80 \pm 10.94$ & & \\
\hline
\end{tabular}

Abbreviations: d.f., degree of freedom; TNM, tumor, lymph node, metastasis. ${ }^{*} P<0.01,{ }^{*} P<0.05$. in T3/T4 tumor staging and III/IV clinical staging compared with lower staging (Table 2).

\section{DISCUSSION}

The regulation of STAT3 transcriptional activity depends mainly on post-translational modifications (PTMs). Phosphorylation at Y705 or S727 is a crucial PTM that triggers STAT3 dimerization, nuclear translocation and maximal transactivation upon cytokine/growth factor stimulation; ${ }^{53}$ however, many other PTMs, including acetylation, methylation, poly- and mono-ubiquitination, are required for STAT3 activation under various contexts. 1,16,17,60,61 SUMOylation, that is, the conjugation of SUMO1 or $2 / 3$ to the substrates, is an important PTM. ${ }^{62}$ SUMO2 and SUMO3 are closely related, referred to as SUMO2/3, whereas SUMO1 shares only partial similarity with SUMO2/3. ${ }^{63}$ SUMOylation of transcription factors have been reported to regulate their transcriptional activity negatively. ${ }^{62,64,65}$ Previously, STAT1 was shown to be unique among the STATs in conjugation with SUMO1. ${ }^{66}$ Herein, we uncover that STAT3 can be conjugated by SUMO2/3 at K451 site, and its nuclear phosphatase TC45 has SIM; SUMO2/3 modification of STAT3 promotes its interaction with TC45. Therefore, SUMOylation of STAT3 negatively regulates its activity by restraining Y705 phosphorylation in the nucleus. Furthermore, this study reveals that the deSUMOylation by SUMO2/3-specific protease SENP3 can enhance STAT3 phosphorylation in the nucleus through hampering its binding with TC45. These findings identify an additional type of PTM to STAT3, which represents a novel and sophisticated regulatory mechanism.

The development and progression of HNC occur through a stepwise and progressive accumulation of genetic or epigenetic alterations mainly due to direct and repeated exposures to environmental carcinogens. ${ }^{67-69}$ Tobacco use via cigarette smoking is a confirmative etiologic factor for HNC. ${ }^{34,35,70}$ Intensive work has been performed in an attempt to understand tobacco carcinogenesis. ${ }^{71-73}$ It is widely accepted that tobacco carcinogens and their metabolites can bind covalently to DNA, causing DNA damage. $^{74,75}$ The development of carcinoma after tobacco use thus has been basically attributed to DNA damage and the consequent genomic instability. ${ }^{76,77}$ Recently, aberrations in a number of genes and signaling pathways have been proposed as

\begin{tabular}{|c|c|c|c|c|}
\hline Characteristics & $\begin{array}{c}\text { Positivity area of } \\
\text { SENP3 (\%) }\end{array}$ & $\begin{array}{c}\text { Positivity area of } \\
\text { p-STAT3 (\%) }\end{array}$ & R-value & P-value \\
\hline \multicolumn{5}{|c|}{ Tobacco smoking } \\
\hline Yes & $29.81 \pm 14.41$ & $33.18 \pm 14.49$ & 0.555 & 0.000 \\
\hline No & $24.15 \pm 13.29$ & $23.26 \pm 14.13$ & 0.374 & 0.015 \\
\hline \multicolumn{5}{|l|}{ Nodal status } \\
\hline No & $26.37 \pm 14.15$ & $28.41 \pm 14.97$ & 0.529 & 0.000 \\
\hline N1-2 & $32.83 \pm 13.74$ & $35.88 \pm 13.69$ & 0.654 & 0.000 \\
\hline \multicolumn{5}{|l|}{ Tumor stage } \\
\hline $\mathrm{T} 1$ & $24.56 \pm 13.53$ & $27.84 . \pm 13.93$ & 0.475 & 0.000 \\
\hline $\mathrm{T} 2$ & $26.64 \pm 11.74$ & $27.26 \pm 14.40$ & 0.480 & 0.000 \\
\hline T3 & $31.41 \pm 15.42$ & $33.86 . \pm 13.23$ & 0.725 & 0.000 \\
\hline T4 & $37.66 \pm 16.40$ & $42.10 \pm 16.80$ & 0.746 & 0.000 \\
\hline \multicolumn{5}{|c|}{ Clinical stage (TNM classification) } \\
\hline 1 & $24.56 \pm 13.53$ & $27.84 \pm 13.93$ & 0.475 & 0.000 \\
\hline II & $23.86 \pm 9.375$ & $25.68 \pm 15.09$ & 0.407 & 0.010 \\
\hline III & $32.07 \pm 15.74$ & $33.78 . \pm 13.38$ & 0.773 & 0.000 \\
\hline IV & $36.64 \pm 14.96$ & $38.96 \pm 15.45$ & 0.573 & 0.003 \\
\hline
\end{tabular}


the key molecular events in HNC pathogenesis, ${ }^{78}$ their association with tobacco products remains unstudied yet. Tobacco exposure has been implicated to correlate with the activation of STAT3 in HNC in vitro and in vivo, ${ }^{22,37,74}$ but questions of where and how tobacco products activate STAT3 in HNC have not been addressed. We herein provide links between tobacco products, STAT3 activation and HNC.

Cigarette smoke contains high concentrations of oxidants, ${ }^{40}$ and has the potential to increase the cellular production of ROS. ${ }^{41,54}$ NNK is a widely recognized tobacco-specific carcinogen $^{51,79,80}$ as well as an oxidant. ${ }^{81,82}$ We demonstrate that exposure of HNC cells to NNK leads to enhanced STAT3 phosphorylation through the generation of ROS, as well as the ROS-dependent induction of SENP3. As cigarette smoking HNC patients may have a cancer microenvironment high in NNK and its toxic metabolites, an upregulated SENP3 protein level is expected; and thus, this type of positive regulation may contribute significantly to persistent STAT3 activation. Interestingly, the SENP3-mediated enhancement of STAT3 activation is based on a pre-existing basal level of the IL-6/JAK/STAT3 signaling. This regulation is used by NNK, and also by additional IL-6, which may mimic the microenvironment deteriorated by inflammatory cytokines and tobacco products (Figure 7). Supported by the relevance of SENP3 and p-STAT3 in laryngeal carcinoma as well as in their clinicopathological parameters, we conclude that this regulatory mechanism may indeed have roles in the development and progression of HNC.

Several fundamental questions in HNC oncology include why some cigarette smokers develop HNC while others do not, that is, what biochemical or genetic factors differentiate smokers with HNC from smokers without HNC, and whether there are better indicators for predicting smokers with the highest risk. ${ }^{83}$ Our findings in this study might provide an explanation for the first question and some clues regarding the second one. A higher HNC susceptibility could not only be determined by genetic defects in DNA damage repair but also be affected by the redox state; higher protein levels of SENP3 induced by oxidative stress may lead to an enhanced activity of STAT3 and its oncogenic function. Therefore, aside from detecting levels of tobacco carcinogen metabolites in serum and urine, detecting the levels of SENP3 and phosphorylated STAT3 in the tissues of pre-cancerous lesions might potentially help to construct a more reasonable and effective biomarker signature.

\section{MATERIALS AND METHODS}

Human biopsies

Laryngeal specimens derived from surgically resected tissues of vocal polyps $(n=12)$, para-carcinoma tissues $(n=52)$, laryngeal dysplasias $(n=10)$, laryngeal carcinoma $(n=159$ : smoking $n=117$, no-smoking $n=42$ ) and the related clinicopathological data were obtained in Ren Ji Hospital following an approved protocol. The patients had not received pre-operative chemotherapy or radiation therapy. The use of human HNC tissue specimens was evaluated and approved by the Ethical Committee of Ren Ji Hospital, Shanghai Jiao Tong University School of Medicine.

\section{Cell culture and treatments}

The cell lines Hep-2, KB and HEK293T were purchased from the American Type Culture Collection (Manassas, VA, USA); HN30 was a gift from the Department of Oral and Maxillofacial-Head and Neck Oncology (Shanghai Jiao Tong University School of Medicine, China). The human HNC cell lines Hep-2 cells were cultured in RPMI-1640 medium (HyClone, Logan, UT, USA). HEK293T cell lines and the human HNC cell lines KB and HN30 and were cultured in Dulbecco's modified Eagle's medium (HyClone). All media were supplemented with $10 \%$ fetal bovine serum (Gibco, Grand Island, NY, USA). When needed, the anti-oxidant NAC (Sigma, St Louis, MO, USA) was pre-administered for $4 \mathrm{~h}$, and the JAK inhibitor (Santa Cruz Biotechnology, Dallas, TX, USA) and anti-IL-6 (InvivoGen, San Diego, CA, USA) were pre-administered for $1 \mathrm{~h}$ before other treatments. Cells were exposed to NNK (Sigma) and IL-6 (R\&D Systems China Co. Ltd., Shanghai, China) at various doses for different times as indicated. Cell transfection was performed using Lipofectamine 2000 (Invitrogen) for plasmid DNA and siRNAs following the manufacturer's instructions. Cells were harvested at $24-48 \mathrm{~h}$ post transfection for protein analyses.

Plasmid mutagenesis and establishment of stable cell lines Flag-STAT3 Lys/Arg and Flag-TC45 Val/Ala mutant constructs were generated by site-directed mutagenesis based on the Flag-STAT3 and Flag-TC45 WT constructs using a QuikChange Mutagenesis Kit (Agilent Technologies, Santa Clara, CA, USA) as described. ${ }^{49}$

To establish the stable STAT3-shRNA (5'UTR) and stable SENP3-shRNA (5'UTR) Hep-2 cell lines, ZsGreen1 co-expressing lentiviral expression vectors were transiently transfected into $293 \mathrm{FT}$ cells. After $72 \mathrm{~h}$, the supernatants were harvested to infect Hep-2 cells with a final concentration of $10 \mu \mathrm{g} / \mathrm{ml}$ polybrene. Finally, the ZsGreen 1-positive cells were sorted
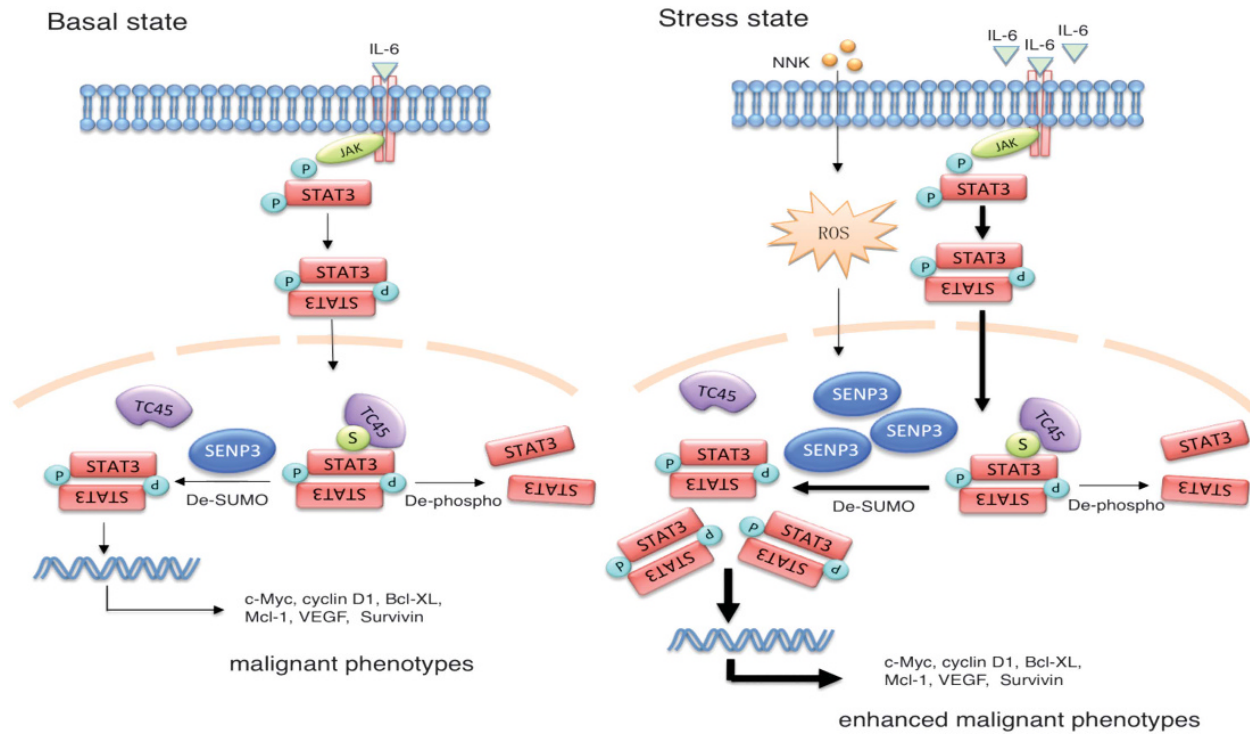

Figure 7. A schema for the roles of SUMOylation and SENP3 in the regulation of STAT3 activation under basal and stressed microenvironments that are deteriorated by cigarette components and excessive IL-6. 
on a FACSAria II flow cytometer (BD Biosciences, San Jose, CA, USA). The primers used in the mutagenesis and establishment of stable cell lines were described in the Supplementary information. The pEGFP-C1-SENP3, pEGFP-C1-SENP3 mutant constructs were previously described. ${ }^{49}$ The siRNA specific for SENP3 and non-specific control siRNA oligonucleotides were synthesized and used as previously described. ${ }^{48}$ The plasmid FlagSTAT3-SUMO3 was purchased from TransheepBio-Tech CO., LTD (Shanghai, China). The primers used in the plasmid mutagenesis and shRNA establishment were described in the Supplementary information.

\section{Immunoblotting}

The methods were as previously described. ${ }^{49}$ The antibodies were described in the Supplementary information.

\section{Flag immunoprecipitation assay}

Transfected cells were lysed in a lysis buffer $(50 \mathrm{~mm}$ Tris- $\mathrm{HCl}, \mathrm{pH} 7.4$, with $150 \mathrm{~mm} \mathrm{NaCl}, 1 \mathrm{~mm}$ EDTA and 1\% Triton X-100). Anti-FLAG M2 Affinity Gel (Cat\#A2220, Sigma) was added to the cell lysates and incubated overnight at $4{ }^{\circ} \mathrm{C}$. The beads were washed four times in the lysis buffer. After the last wash, Flag-tagged proteins were eluted in elution buffer (lysis buffer, cocktail (Roche, Diagnostics GmbH, Mannheim, Germany), 20 mM NEM (Sigma)) and then subjected to IB.

\section{Co-IP assay}

Cells were lysed and sonicated in RIPA buffer (Thermo Scientific, Pittsburgh, PA, USA) at $4{ }^{\circ} \mathrm{C}$ for $30 \mathrm{~min}$, then centrifuged at $13000 \mathrm{~g}$ at $4{ }^{\circ} \mathrm{C}$ for another $30 \mathrm{~min}$. The cell lysates were pre-cleared by adding $40 \mu \mathrm{l}$ Protein-A/G agarose beads (Cat\#IP05, Calbiochem, Temecula, CA, USA) per $1 \mathrm{ml}$ and incubating at $4{ }^{\circ} \mathrm{C}$ for $30 \mathrm{~min}$. The protein-A/G beads were then removed by centrifugation. Specific antibodies were mixed with the supernatants overnight at $4{ }^{\circ} \mathrm{C}$. Protein- $\mathrm{A} / \mathrm{G}$ agarose beads were added to the lysate, and the mixture was incubated under shaking for $4 \mathrm{~h}$ at $4^{\circ} \mathrm{C}$. The beads were washed three times, mixed with loading buffer and examined by SDS-PAGE and IB analyses.

\section{ROS detection}

2',7'-Dichlorofluorescin diacetate (DCFH-DA; Sigma) was used as an ROScapturing reagent as previously described. ${ }^{84}$

\section{FRET imaging}

Hep-2 cells transfected with Flag-tagged WT STAT3 or STAT3-SUMO3 fusion vector for $24 \mathrm{~h}$, then grown on coverslips were fixed in $4 \%$ paraformaldehyde for $10 \mathrm{~min}$ before permeabilization with $0.3 \%$ Triton X-100 in phosphate washed with PBS and incubated with $2 \%$ BSA containing primary antibodies against Flag and TCPTP at $4{ }^{\circ} \mathrm{C}$ overnight. The monolayers were then washed with PBS and incubated with appropriate secondary antibodies in PBS at $37^{\circ} \mathrm{C}$ for $2 \mathrm{~h}$. Alexa Fluor 488-labelled goat anti-rabbit antibodies and Alexa Fluor 546-labelled goat anti-mouse antibodies were obtained from Molecular Probes (Eugene, OR, USA) and Invitrogen. Cells were washed again with PBS, counterstained with DAPI (Beyotime, Shanghai, China) and then used for observation.

FRET was performed on Zeiss LSM 710 laser scanning confocal microscope (Carl Zeiss, Inc., Oberkochen, Germany) with a Plan Apochromat $\times 63.0,1.4 \mathrm{NA}$ oil-immersion, differential interference contrast objective, and a 488-nm argon laser. Images were scanned in $1024 \times 1024$ format, $1.58 \mu$ s pixel dwell, 12-bit depth, $4 \times$ line average and $3.0 \mu \mathrm{m}$ pinhole. Sensitized emission method was performed using 'normalized FRET (N-FRET) measurement' function in Zeiss microscope software ZEN 2011 (Carl Zeiss, Inc.). Three channel of images were recorded as following: donor, $488 \mathrm{~nm}$ laser excitation and 490-582 nm filter; acceptor, $532 \mathrm{~nm}$ laser excitation and 552-672 nm filter; FRET, $488 \mathrm{~nm}$ laser excitation and 552-672 nm filter. Individual donor and acceptor images were analyzed and loaded into the two-positive images and calculated the final N-FRET images.

\section{Cell viability assessment by Cell Counting Kit (CCK8)}

According to the manufacturer's protocol, CCK8 (Peptide Institute Inc., Osaka, Japan) solution was added to each sample and incubated for $1 \mathrm{~h}$. The absorbance of solution was recorded at $450 \mathrm{~nm}$ with a microplate reader (Thermo Fisher Scientific Inc., Waltham, MA, USA).
Transwell migration assay

Transwell assays were performed with $8.0 \mu \mathrm{m}$ pore size 24-well Transwell plates (Corning, Corelle, NY, USA) as previously described. ${ }^{50}$ After $10 \mathrm{~h}$ at $37^{\circ} \mathrm{C}$, cells on the lower surface of the membrane were fixed with paraformaldehyde and stained with crystal violet. The number of cells migrating was counted under a light microscope $(\times 20$, three random fields per well).

\section{Real-time qPCR}

Real-time qPCR was conducted on the ABI Prism 7500 system using SYBR Green (Roche, Diagnostics $\mathrm{GmbH}$ ) according to the manufacturer's instructions. The primers used in the real-time PCR were described in the Supplementary information.

\section{Soft agar colony formation assay}

The method was described in the Supplementary information.

Nuclear and cytoplasmic fractionation

The method was described in the Supplementary information.

\section{Immunohistochemistry}

The paraformaldehyde-fixed and paraffin-embedded sections of human laryngeal specimens were archived pathological specimens from the Ren Ji Hospitals and obtained after securing institutional approval of the protocol. The immunohistochemistry for the SENP3 and pY705-STAT3 was performed using serial sections as previously described. ${ }^{85}$ The ratio of the positive areas for SENP3 and pY705-STAT3 was quantified by the Zeiss KS400 software (Carl Zeiss, Inc.). The mean optical densities of SENP3 and pY705-STAT3 were quantified using the software Image J (National Institutes of Health, USA).

\section{SUMOylation site and SIM predictions}

Prediction of SUMOylation probability and sites was carried out using the open software SUMOplot Analysis Program. Prediction for SIM sites was carried out using the open software GPS-SBM 1.0.

\section{Statistics analysis}

Statistical analyses Microsoft Excel (San Francisco, CA, USA) or GraphPad Prism (La Jolla, CA, USA) was used to analyze data. Non-parametric (Kruskal-Wallis, Mann-Whitney) tests were used to compare the difference of the clinicopathological features and SENP3 expression. The relationship between the expression of SENP3 and pY705-STAT3 was examined by the Spearman Rank correlation coefficient. We considered $P<0.05$ to be statistically significant. All statistical analyses were expressed as mean \pm s.d. No sample was excluded from the analysis.

\section{CONFLICT OF INTEREST}

The authors declare no conflict of interest.

\section{ACKNOWLEDGEMENTS}

This work was supported by grants from the National Ministry of Science and Technology of China (2013CB910900), the National Natural Science Foundation of China (31230037) and Shanghai Municipal Science and Technology Commission (11DZ2260200, 13ZR1425300). The plasmid Flag-STAT3 and Flag-TC45 were gifts of Dr Guoliang Xu (Institute of Biochemistry and Cell Biology, Chinese Academy of Sciences, China)

\section{REFERENCES}

1 Zhong Z, Wen Z, Darnell JE Jr. STAT3: a STAT family member activated by tyrosine phosphorylation in response to epidermal growth factor and interleukin-6. Science 1994; 264: 95-98.

2 Levy DE, Darnell JE. Signalling: Stats: transcriptional control and biological impact. Nat Rev Mol Cell Biol 2002; 3: 651-662.

3 Mantovani A, Allavena P, Sica A, Balkwill F. Cancer-related inflammation. Nature 2008; 454: 436-444. 
4 Becker C, Fantini MC, Schramm C, Lehr HA, Wirtz S, Nikolaev A et al. TGF-beta suppresses tumor progression in colon cancer by inhibition of IL- 6 trans-signaling. Immunity 2004; 21: 491-501.

5 Berishaj M, Gao SP, Ahmed S, Leslie K, Al-Ahmadie H, Gerald WL et al. STAT3 is tyrosine-phosphorylated through the interleukin-6/glycoprotein 130/Janus kinase pathway in breast cancer. Breast Cancer Res 2007; 9: R32.

6 Morikawa T, Baba Y, Yamauchi M, Kuchiba A, Nosho K, Shima K et al. STAT3 expression, molecular features, inflammation patterns, and prognosis in a database of 724 colorectal cancers. Clin Cancer Res 2011; 17: 1452-1462.

7 Kim D-Y, Cha S-T, Ahn D-H, Kang H-Y, Kwon C-I, Ko K-H et al. STAT3 expression in gastric cancer indicates a poor prognosis. J Gastroenterol Hepatol 2009; 24 646-651.

8 Lou W, Ni Z, Dyer K, Tweardy DJ, Gao AC. Interleukin-6 induces prostate cancer cell growth accompanied by activation of STAT3 signaling pathway. Prostate 2000; 42: 239-242.

9 Masuda M, Suzui M, Yasumatu R, Nakashima T, Kuratomi Y, Azuma K et al. Constitutive activation of signal transducers and activators of transcription 3 correlates with Cyclin D1 overexpression and may provide a novel prognostic marker in head and neck squamous cell carcinoma. Cancer Res 2002; 62: 3351-3355.

$10 \mathrm{Yu}$ H, Pardoll D, Jove R. STATs in cancer inflammation and immunity: a leading role for STAT3. Nat Rev Cancer 2009; 9: 798-809.

11 Bromberg J, Wang TC. Inflammation and cancer: IL-6 and STAT3 complete the link. Cancer Cell 2009; 15: 79-80.

12 Li N, Grivennikov Sl, Karin M. The Unholy Trinity: inflammation, cytokines, and STAT3 shape the cancer microenvironment. Cancer Cell 2011; 19: 429-431.

13 Groner B, Lucks P, Borghouts C. The function of STAT3 in tumor cells and their microenvironment. Semin Cell Dev Biol 2008; 19: 341-350.

14 Pilati C, Amessou M, Bihl MP, Balabaud C, Van Nhieu JT, Paradis V et al. Somatic mutations activating STAT3 in human inflammatory hepatocellular adenomas. J Exp Med 2011; 208: 1359-1366.

15 Rokavec M, Öner MG, Li H, Jackstadt R, Jiang L, Lodygin D et al. IL-6 R/STAT3/ miR-34a feedback loop promotes EMT-mediated colorectal cancer invasion and metastasis. J Clin Invest 2014; 124: 1853-1867.

16 Yuan Z-L, Guan Y-J, Chatterjee D, Chin YE. STAT3 dimerization regulated by reversible acetylation of a single lysine residue. Science 2005; 307: 269-273.

17 Yang J, Huang J, Dasgupta M, Sears N, Miyagi M, Wang B et al. Reversible methylation of promoter-bound STAT3 by histone-modifying enzymes. Proc Nat/ Acad Sci USA 2010; 107: 21499-21504.

18 Chen X, Ying Z, Lin X, Lin H, Wu J, Li M et al. Acylglycerol kinase augments JAK2/STAT3 signaling in esophageal squamous cells. J Clin Invest 2013; 123: 2576-2589.

19 Zhang $\mathrm{P}$, Zhao Y, Zhu X, Sedwick D, Zhang X, Wang Z. Cross-talk between phospho-STAT3 and PLC 1 plays a critical role in colorectal tumorigenesis. Mol Cancer Res 2011; 9: 1418-1428.

20 He Q-Y, Yi H-M, Yi H, Xiao T, Qu J-Q, Yuan L et al. Reduction of RKIP expression promotes nasopharyngeal carcinoma invasion and metastasis by activating STAT3 signaling. Oncotarget 2015; 6: 16422-16436.

21 Song J, Grandis JR. STAT signaling in head and neck cancer. Oncogene 2000; 19 : 2489-2495.

22 Nagpal JK, Mishra R, Das BR. Activation of Stat-3 as one of the early events in tobacco chewing-mediated oral carcinogenesis. Cancer 2002; 94: 2393-2400.

23 Masuda M, Wakasaki T, Suzui M, Toh S, Joe AK, Weinstein IB. STAT3 orchestrates tumor development and progression: the Achilles' heel of head and neck cancers? Curr Cancer Drug Targets 2010; 10: 117-126.

24 Leeman RJ, Lui VWY, Grandis JR. STAT3 as a therapeutic target in head and neck cancer. Expert Opin Biol Ther 2006; 6: 231-241.

25 Liu T, Peng H, Zhang M, Deng Y, Wu Z. Cucurbitacin B, a small molecule inhibitor of the STAT3 signaling pathway, enhances the chemosensitivity of laryngeal squamous cell carcinoma cells to cisplatin. Eur J Pharmacol 2010; 641: 15-22.

26 Gao LF, Wen L, Yu H, Zhang L, Meng Y, Shao YT et al. Knockdown of STAT3 expression using RNAi inhibits growth of laryngeal tumors in vivo. Acta Pharmacol Sin 2006; 27: 347-352.

27 Gao LF, Xu DQ, Wen LJ, Zhang XY, Shao YT, Zhao XJ. Inhibition of STAT3 expression by siRNA suppresses growth and induces apoptosis in laryngeal cancer cells. Acta Pharmacol Sin 2005; 26: 377-383.

28 Xi S, Gooding WE, Grandis JR. In vivo antitumor efficacy of STAT3 blockade using a transcription factor decoy approach: implications for cancer therapy. Oncogene 2004; 24: 970-979.

29 Grandis JR, Drenning SD, Chakraborty A, Zhou MY, Zeng Q, Pitt AS et al. Requirement of STAT3 but not Stat1 activation for epidermal growth factor receptor- mediated cell growth In vitro. J Clin Invest 1998; 102: 1385-1392.

30 Grandis JR, Drenning SD, Zeng Q, Watkins SC, Melhem MF, Endo S et al. Constitutive activation of STAT3 signaling abrogates apoptosis in squamous cell carcinogenesis in vivo. Proc Natl Acad Sci USA 2000; 97: 4227-4232.
31 Lee TL. Epigenetic modification of SOCS-1 differentially regulates STAT3 activation in response to interleukin-6 receptor and epidermal growth factor receptor signaling through JAK and/or MEK in head and neck squamous cell carcinomas. Mol Cancer Ther 2006; 5: 8-19.

32 Sriuranpong V, Park Jl, Amornphimoltham P, Patel V, Nelkin BD, Gutkind JS Epidermal growth factor receptor-independent constitutive activation of STAT3 in head and neck squamous cell carcinoma is mediated by the autocrine/paracrine stimulation of the interleukin 6/gp130 cytokine system. Cancer Res 2003; 63: 2948-2956.

33 Squarize $\mathrm{CH}$, Castilho RM, Sriuranpong V, Pinto Jr DS, Gutkind JS. Molecular cross-talk between the NFkappaB and STAT3 signaling pathways in head and neck squamous cell carcinoma. Neoplasia 2006; 8: 733-746.

34 Hashibe M, Brennan P, Benhamou S, Castellsague X, Chen C, Curado MP et al. Alcohol drinking in never users of tobacco, cigarette smoking in never drinkers, and the risk of head and neck cancer: pooled analysis in the International Head and Neck Cancer Epidemiology Consortium. J Natl Cancer Inst 2007; 99: 777-789.

35 Lewin F, Norell SE, Johansson H, Gustavsson P, Wennerberg J, Biörklund A et al. Smoking tobacco, oral snuff, and alcohol in the etiology of squamous cell carcinoma of the head and neck. Cancer 1998; 82: 1367-1375.

36 Blot WJ, McLaughlin JK, Winn DM, Austin DF, Greenberg RS, Preston-Martin S et al. Smoking and drinking in relation to oral and pharyngeal cancer. Cancer Res 1988; 48: 3282-3287.

37 Macha MA, Matta A, Chauhan SS, Siu KWM, Ralhan R. Guggulsterone (GS) inhibits smokeless tobacco and nicotine-induced NF-KB and STAT3 pathways in head and neck cancer cells. Carcinogenesis 2011; 32: 368-380.

38 Liu X. STAT3 activation inhibits human bronchial epithelial cell apoptosis in response to cigarette smoke exposure. Biochem Biophys Res Commun 2007; 353: 121-126.

39 Chen RJ, Ho YS, Guo HR, Wang YJ. Rapid activation of STAT3 and ERK1/2 by nicotine modulates cell proliferation in human bladder cancer cells. Toxicol Sci 2007; 104: 283-293.

40 Csiszar A, Podlutsky A, Wolin MS, Losonczy G, Pacher P, Ungvari Z. Oxidative stress and accelerated vascular aging: implications for cigarette smoking. Front Biosci (Landmark Ed) 2009; 14: 3128-3144.

41 Pryor WA, Prier DG, Church DF. Electron-spin resonance study of mainstream and sidestream cigarette smoke: nature of the free radicals in gas-phase smoke and in cigarette tar. Environ Health Perspect 1983; 47: 345-355.

42 Zang LY, Stone K, Pryor WA. Detection of free radicals in aqueous extracts of cigarette tar by electron spin resonance. Free Radic Biol Med 1995; 19: 161-167.

43 Pryor WA, Stone K. Oxidants in cigarette smoke. Radicals, hydrogen peroxide, peroxynitrate, and peroxynitrite. Ann NY Acad Sci 1993; 686: 12-27.

$44 \mathrm{Ng}$ IHW, Yeap YYC, Ong LSR, Jans DA, Bogoyevitch MA. Oxidative stress impairs multiple regulatory events to drive persistent cytokine-stimulated STAT3 phosphorylation. Biochim Biophys Acta 2014; 1843: 483-494.

45 Qu Y, Oyan AM, Liu R, Hua Y, Zhang J, Hovland R et al. Generation of prostate tumor-initiating cells is associated with elevation of reactive oxygen species and IL-6/STAT3 signaling. Cancer Res 2013; 73: 7090-7100.

46 Bito T, Sumita N, Masaki T, Shirakawa T, Ueda M, Yoshiki R et al. Ultraviolet light induces STAT3 activation in human keratinocytes and fibroblasts through reactive oxygen species and DNA damage. Exp Dermatol 2010; 19: 654-660.

47 Huang C, Han Y, Wang Y, Sun X, Yan S, Yeh ETH et al. SENP3 is responsible for HIF-1 transactivation under mild oxidative stress via p300 de-SUMOylation. EMBO J 2009; 28: 2748-2762.

48 Han $Y$, Huang $C$, Sun $X$, Xiang $B$, Wang $M$, Yeh ETH et al. SENP3-mediated de-conjugation of SUMO2/3 from promyelocytic leukemia is correlated with accelerated cell proliferation under mild oxidative stress. J Biol Chem 2010; 285 12906-12915.

49 Yan S, Sun X, Xiang B, Cang H, Kang X, Chen $Y$ et al. Redox regulation of the stability of the SUMO protease SENP3 via interactions with CHIP and Hsp90. EMBO J 2010; 29: 3773-3786.

50 Ren Y, Liu K, Wang M, Yu Y, Yang K, Chen Q et al. De-SUMOylation of FOXC2 by SENP3 promotes the epithelial-mesenchymal transition in gastric cancer cells. Oncotarget 2014; 5: 7093-7104.

51 Hecht SS. Tobacco carcinogens, their biomarkers and tobacco-induced cancer. Nat Rev Cancer 2003; 3: 733-744.

52 Schindler C, Darnell JE. Transcriptional responses to polypeptide ligands: the JAK-STAT pathway. Annu Rev Biochem 1995; 64: 621-651.

53 Wen Z, Zhong Z, Darnell JE Jr. Maximal activation of transcription by stat and STAT3 requires both tyrosine and serine phosphorylation. Cell 1995; 82: 241-250.

54 Pryor WA, Stone K, Zang LY, Bermúdez E. Fractionation of aqueous cigarette tar extracts: fractions that contain the tar radical cause DNA damage. Chem Res Toxicol 1998; 11: 441-448.

55 Yeh E. SUMOylation and De-SUMOylation: wrestling with life's processes. J Biol Chem 2009; 284: 8223-8227. 
56 Chen W, Daines MO, Khurana Hershey GK. Turning off signal transducer and activator of transcription (STAT): The negative regulation of STAT signaling. J Allergy Clin Immunol 2004; 114: 476-489.

57 Yamamoto T, Sekine Y, Kashima K, Kubota A, Sato N, Aoki N et al. The nuclear isoform of protein-tyrosine phosphatase TC-PTP regulates interleukin-6-mediated signaling pathway through STAT3 dephosphorylation. Biochem Biophys Res Commun 2002; 297: 811-817.

58 Johnston PA, Grandis JR. STAT3 signaling: anticancer strategies and challenges. Mol Interv 2011; 11: 18-26.

59 Wilkinson KA, Henley JM. Mechanisms, regulation and consequences of protein SUMOylation. Biochem J 2010; 428: 133-145.

60 Ulane CM, Rodriguez JJ, Parisien JP, Horvath CM. STAT3 ubiquitylation and degradation by mumps virus suppress cytokine and oncogene signaling. J Virol 2003; 77: 6385-6393.

61 Perry E, Tsruya R, Levitsky P, Pomp O, Taller M, Weisberg S et al. TMF/ARA160 is a $\mathrm{BC}$-box-containing protein that mediates the degradation of STAT3. Oncogene 2004; 23: 8908-8919.

62 Geiss-Friedlander R, Melchior F. Concepts in sumoylation: a decade on. Nat Rev Mol Cell Biol 2007; 8: 947-956.

63 Yeh ET, Gong L, Kamitani T. Ubiquitin-like proteins: new wines in new bottles. Gene 2000; 248: 1-14.

64 Gill G. Something about SUMO inhibits transcription. Curr Opin Genet Dev 2005; 15: $536-541$.

65 Girdwood DWH, Tatham MH, Hay RT. SUMO and transcriptional regulation. Semin Cell Dev Biol 2004; 15: 201-210.

66 Droescher M, Begitt A, Marg A, Zacharias M, Vinkemeier U. Cytokine-induced paracrystals prolong the activity of signal transducers and activators of transcription (STAT) and provide a model for the regulation of protein solubility by Small Ubiquitin-like Modifier (SUMO). J Biol Chem 2011; 286: 18731-18746.

67 Hunter KD, Parkinson EK, Harrison PR. Profiling early head and neck cancer. Nat Rev Cancer 2005; 5: 127-135.

68 Quon H, Liu FF, Cummings BJ. Potential molecular prognostic markers in head and neck squamous cell carcinomas. Head Neck 2001; 23: 147-159.

69 Le Q-T, Giaccia AJ. Therapeutic exploitation of the physiological and molecular genetic alterations in head and neck cancer. Clin Cancer Res 2003; 9: 4287-4295.

70 Iwamoto H. An epidemiological study of laryngeal cancer in Japan. Laryngoscope 1975; 85: 1162-1172.

71 Parfenov M, Pedamallu CS, Gehlenborg N, Freeman SS, Danilova L, Bristow CA et al. Head and neck cancer [Internet]. Proc Natl Acad Sci USA 2014; 111: 15544-15549.

72 Takahashi H, Ogata H, Nishigaki R, Broide DH, Karin M. Tobacco smoke promotes lung tumorigenesis by triggering IKKß- and JNK1-dependent inflammation. Cancer Cell 2010; 17: 89-97.

73 Chen R-J, Chang LW, Lin P, Wang Y-J. Epigenetic effects and molecular mechanisms of tumorigenesis induced by cigarette smoke: an overview. J Oncol 2011; 2011: 1-14.
74 Arredondo J. Receptor-mediated tobacco toxicity: cooperation of the Ras/Raf-1/ MEK1/ERK and JAK-2/STAT-3 pathways downstream of alpha7 nicotinic receptor in oral keratinocytes. FASEB J 2006; 20: 2093-2101.

75 Hainaut $P$, Pfeifer GP. Patterns of $p 53 \mathrm{G} \rightarrow T$ transversions in lung cancers reflect the primary mutagenic signature of DNA-damage by tobacco smoke. Carcinogenesis 2001; 22: 367-374.

76 Pfeifer GP, Denissenko MF, Olivier M, Tretyakova N, Hecht SS, Hainaut P. Tobacco smoke carcinogens, DNA damage and p53 mutations in smoking-associated cancers. Oncogene 2002; 21: 7435-7451.

77 Hittelman WN. Genetic instability in epithelial tissues at risk for cancer. Ann NY Acad Sci 2001; 952: 1-12.

78 Rothenberg SM, Ellisen LW. The molecular pathogenesis of head and neck squamous cell carcinoma. J Clin Invest 2012; 122: 1951-1957.

79 Huang R-Y, Li M-Y, Hsin MKY, Underwood MJ, Ma LT, Mok TSK et al. 4-Methylnitrosamino-1-3-pyridyl-1-butanone (NNK) promotes lung cancer cell survival by stimulating thromboxane A2 and its receptor. Oncogene 2010; 30: 106-116.

80 Hecht SS. Biochemistry, biology, and carcinogenicity of tobacco-specific N-nitrosamines. Chem Res Toxicol 1998; 11: 559-603.

81 Ye YN, Liu ESL, Shin VY, Wu WKK, Cho CH. The modulating role of nuclear factorkappaB in the action of alpha7-nicotinic acetylcholine receptor and cross-talk between 5-lipoxygenase and cyclooxygenase-2 in colon cancer growth induced by 4-(N-methyl-N-nitrosamino)-1-(3-pyridyl)-1-butanone. J Pharmacol Exp Ther 2004; 311: 123-130.

82 Kim PM, Wells PG. Genoprotection by UDP-glucuronosyltransferases in peroxidase-dependent, reactive oxygen species-mediated micronucleus Initiation by the carcinogens 4-(Methylnitrosamino)-1-(3-pyridyl)-1-butanone and benzo[a] pyrene. Cancer Res 1996; 56: 1526-1532.

83 Khariwala SS, Hatsukami D, Hecht SS. Tobacco carcinogen metabolites and DNA adducts as biomarkers in Head and Neck cancer: Potential screening tools and prognostic indicators. Head Neck 2011; 34: 441-447.

84 Yang J, Li H, Chen Y-Y, Wang X-J, Shi G-Y, Hu Q-S et al. Anthraquinones sensitize tumor cells to arsenic cytotoxicity in vitro and in vivo via reactive oxygen species-mediated dual regulation of apoptosis. Free Radic Biol Med 2004; 37: 2027-2041.

85 Sang J, Yang K, Sun Y, Han Y, Cang H, Chen $\mathrm{Y}$ et al. SUMO2 and SUMO3 transcription is differentially regulated by oxidative stress in an Sp1dependent manner. Biochem J 2011; 435: 489-498.

This work is licensed under a Creative Commons AttributionNonCommercial-NoDerivs 4.0 International License. The images or other third party material in this article are included in the article's Creative Commons license, unless indicated otherwise in the credit line; if the material is not included under the Creative Commons license, users will need to obtain permission from the license holder to reproduce the material. To view a copy of this license, visit http:// creativecommons.org/licenses/by-nc-nd/4.0/

Supplementary Information accompanies this paper on the Oncogene website (http://www.nature.com/onc) 